\title{
New Numerical Results for the Surface Quasi-Geostrophic Equation
}

\author{
Peter Constantin • Ming-Chih Lai · Ramjee Sharma • \\ Yu-Hou Tseng $\cdot$ Jiahong Wu
}

Received: 18 October 2010 / Revised: 23 January 2011 / Accepted: 31 January 2011

(C) Springer Science+Business Media, LLC 2011

\begin{abstract}
The question whether classical solutions of the surface quasi-geostrophic (SQG) equation can develop finite-time singularities remains open. This paper presents new numerical computations of the solutions to the SQG equation corresponding to several classes of initial data previously proposed by Constantin et al. (Nonlinearity 7:1495-1533, 1994). By parallelizing the serial pseudo-spectral codes through slab decompositions and applying suitable filters, we are able to simulate these solutions with great precision and on large time intervals. These computations reveal detailed finite-time behavior, large-time asymptotics and key parameter dependence of the solutions and provide information for further investigations on the global regularity issue concerning the SQG equation.
\end{abstract}

Keywords Global regularity · Parallel computation · Surface quasi-geostrophic equation

\section{Introduction}

This work presents new numerical results for the surface quasi-geostrophic (SQG) equation

$$
\partial_{t} \theta+u \cdot \nabla \theta+\kappa(-\Delta)^{\alpha} \theta=0,
$$

\footnotetext{
P. Constantin

Department of Mathematics, University of Chicago, Chicago, IL 60637, USA

e-mail: const@cs.uchicago.edu
}

M.-C. Lai · Y.-H. Tseng

Department of Applied Mathematics, National Chiao Tung University, Hsinchu, Taiwan 30010

M.-C. Lai

e-mail: mclai@math.nctu.edu.tw

R. Sharma $(\bowtie)$

Department of Mathematics, Georgia Perimeter College, Atlanta, GA 30338, USA

e-mail: ramjee.sharma@gpc.edu

J. Wu

Department of Mathematics, Oklahoma State University, Stillwater, OK 74078, USA

e-mail: jiahong@math.okstate.edu 
where $\kappa \geq 0$ and $\alpha>0$ are parameters, $\theta=\theta\left(x_{1}, x_{2}, t\right)$ is a scalar representing the potential temperature, and $u=\left(u_{1}, u_{2}\right)$ is the velocity field determined from $\theta$ by the stream function $\psi$ via the auxiliary relations

$$
\left(u_{1}, u_{2}\right)=\left(-\partial_{x_{2}} \psi, \partial_{x_{1}} \psi\right), \quad(-\Delta)^{\frac{1}{2}} \psi=-\theta
$$

Using the notation $\Lambda \equiv(-\Delta)^{\frac{1}{2}}$ and $\nabla^{\perp} \equiv\left(-\partial_{x_{2}}, \partial_{x_{1}}\right)$, the relations in (1.2) can be combined into

$$
u=\nabla^{\perp} \Lambda^{-1} \theta=\left(-\mathcal{R}_{2} \theta, \mathcal{R}_{1} \theta\right),
$$

where $\mathcal{R}_{1}$ and $\mathcal{R}_{2}$ are the usual Riesz transforms. The spatial domain concerned here is the periodic box $\mathbf{T}^{2}$.

The general 3D quasi-geostrophic equations, first derived by J.G. Charney in the 1940s, have been very successful in describing major features of large-scale motions in the atmosphere and the oceans in the midlatitudes (see e.g. [33, 45, 54]). The dynamics of these $3 \mathrm{D}$ equations with uniform potential vorticity reduces to the SQG equation, modeling the evolution of buoyancy, or potential temperature, on the 2D horizontal boundaries [2, 15, 35]. The inviscid SQG equation ((1.1) with $\kappa=0)$ is useful in modeling atmospheric phenomena such as the frontogenesis, the formation of strong fronts between masses of hot and cold air $[15,54]$. In addition, the inviscid SQG equation is a significant example of 2D active scalars and some of its distinctive features have made it an important testbed for turbulence theories $[2,39]$. When $\kappa>0$, the SQG equation takes into account the dissipation generated by a fractional Laplacian. The SQG equation with $\kappa>0$ and $\alpha=\frac{1}{2}$ arises in geophysical studies of strongly rotating fluids (see $[46,54]$ and references therein).

The numerical study presented here is aimed at the fundamental issue of whether solutions of the SQG equation emanating from smooth data can develop finite-time singularities. This issue has recently attracted the interest of many researchers and important progress has been made (see e.g. [1, 3-7, 9-19, 21-32, 36, 38-44, 47-52, 55-73]). We summarize some of the theoretical results here in order to put our computations in a suitable perspective. For the inviscid SQG equation, it has been shown that $L^{2}$-weak solutions are global in time and physically reasonable smooth solutions are at least local in time (see e.g. $[15,55,67])$. In addition, classical solutions whose level curves exhibit certain geometric configurations do not develop finite-time singularities [8, 22, 23]. The global regularity issue for general initial data remains open. For the dissipative SQG equation, $\alpha=\frac{1}{2}$ appears to be a critical index. In the subcritical case when $\alpha>\frac{1}{2}$, the dissipation is sufficient to control the nonlinearity and the global regularity is a consequence of global a priori bound (see $[17,55]$ ). In the critical case when $\alpha=\frac{1}{2}$, the global regularity issue is more delicate. Constantin, Córdoba and $\mathrm{Wu}$ established the global regularity of classical solutions corresponding to data with $L^{\infty}$-norm comparable to $\kappa$ [13]. The global regularity for general data was recently obtained by Kiselev, Nazarov and Volberg for the periodic case [41] and by Caffarelli and Vasseur for the whole space case [4]. The mystery in the supercritical case $\alpha<\frac{1}{2}$ is only partially uncovered at this moment. The results in $[18,19,31]$ imply that any solution of the supercritical SQG equation can only develop a finite time singularity in the regularity window between $L^{\infty}$ and $C^{\delta}$ with $\delta<1-2 \alpha$.

Numerical experiments have become an indispensable tool in studying the global regularity issue on the SQG equation. In the pioneering work on the inviscid SQG equation [15], Constantin, Majda and Tabak computed solutions corresponding to the following initial data 
Type I: $\quad \theta(x, 0)=\sin \left(x_{1}\right) \sin \left(x_{2}\right)+\cos \left(x_{2}\right)$,

Type II: $\quad \theta(x, 0)=-\left(\cos \left(2 x_{1}\right) \cos \left(x_{2}\right)+\sin \left(x_{1}\right) \sin \left(x_{2}\right)\right)$,

Type III: $\quad \theta(x, 0)=\cos \left(2 x_{1}\right) \cos \left(x_{2}\right)+\sin \left(x_{1}\right) \sin \left(x_{2}\right)+\cos \left(2 x_{1}\right) \sin \left(3 x_{2}\right)$.

These initial data are combinations of the first several lowest eigenmodes and represent the simplest type of smooth initial data with nonlinear behavior. They found that the corresponding solutions of these data behave differently. Equation (1.4) leads to a front formation with a hyperbolic saddle, while (1.5) leads to a front formation with an elliptic center. Equation (1.6) is a combination of (1.4) and (1.5). The solution emanating from (1.4) appears to exhibit the most singular behavior. Its gradient grows so fast that $\max _{x \in \mathbf{T}^{2}}|\nabla \theta|$ can be fitted by a finite-time singular profile

$$
\max _{x \in \mathbf{T}^{2}}|\nabla \theta(\cdot, t)| \sim\left(T^{*}-t\right)^{-1.66}
$$

for $T * \cong 8.25$. Further computations in [52] and [16] imply that $\max _{x \in \mathbf{T}^{2}}|\nabla \theta|$ can also be fitted by a double exponential function of $t$. The analytic work of Córdoba [20] appears to confirm the double exponential growth conclusion.

The numerical results presented in [15, 52] and [16] only recorded the behavior of the solutions before the time $t$ reaches 8 , due to the lack of sufficient resolution. One goal here is to compute the solutions on much longer time intervals with sufficient resolutions and thus to completely understand the behavior of these solutions. To fully resolve the solutions numerically, we parallelize the serial pseudo-spectral codes using slab decomposition and perform the computations on a cluster of 128 machines with several mesh sizes: $512 \times 512$, $1024 \times 1024,2048 \times 2048$ and $4096 \times 4096$. For grid points $2048 \times 2048$ and $4096 \times 4096$, the computations are well resolved for all time and our computations stopped around $t=20$ when the large-time behavior of solutions become apparent.

Our numerical experiments are divided into two groups with the first devoted to the inviscid SQG equation and the second to the SQG equation with dissipation. For the inviscid SQG equation, we compute the solutions corresponding to the data in (1.4), (1.5) and (1.6). For each solution $\theta$, we plot the level curves of $\theta$ at various times to record its time evolution. To determine whether $|\nabla \theta|$ can grow without a bound for the data in (1.4), we plot the snapshots of the spatial regions with large $|\nabla \theta|$ and keep track of the time evolution of the maximum of $|\nabla \theta|$. As the log-log plot of $\max _{x \in \mathbf{T}^{2}}|\nabla \theta|$ verses $t$ indicates, the maximum of $|\nabla \theta|$ initially grows rapidly but starts decreasing after it reaches the peak around $t=13.5$. In the case of initial data (1.4) we found that, after the formation of the strong hyperbolic saddle front, its evolution generates the formation of a nearby strong front, and the maximum gradient is attained in a situation in which the hyperbolic saddle is not strong, but rather is found in the anti-parallel configuration of the second front, around time 13.5. The reason for subsequent decay is not clear, and should be the subject of further investigations. We remark that, as far as computed, after the gradient decay sets in, there is no re-formation of organized, strong fronts. More details of these plots are described in the first subsection of Sect. 3 .

The numerical experiments for the dissipative SQG equation ((1.1) with $\kappa>0)$ test how $\alpha$ and $\kappa$ affect the regularity of the solutions. In particular, we intend to numerically check if the solutions in the supercritical case $\alpha<\frac{1}{2}$ can develop finite singularities. More precisely, we compute the solutions of (1.1) corresponding to the data in (1.4), (1.5) and (1.6) and to a range of $\alpha$ 's and $\kappa$ 's. Special attention is paid to the behavior of solutions for $\alpha$ near the 
supposedly critical index $\frac{1}{2}$. As the contour plots indicate, the solutions do not experience any drastic changes when $\alpha$ crosses $\frac{1}{2}$. We present the details of our results in the second subsection of Sect. 3. These results are reaffirmed by a very recent work of Ohkitani and Sakajo [53]. Their results also imply that $\alpha=\frac{1}{2}$ is not critical from a numerical point of view. In the case when $\alpha<\frac{1}{2}$, the work of Ohkitani and Sakajo reported under-resolution after $t=9.5$, even though they used a much higher number of grid points. Our computations around this time do not show any under-resolution and can be continued after that time. Our understanding is that the high order exponential filter that we used in our computations keeps the solutions from being under resolved for a longer time.

Although we still use the pseudo-spectral scheme, it is parallelized together with the parallelized fast Fourier transform and inverse fast Fourier transform routines. More precisely, we use the MPI FFTW (Fast Fourier Transform in the West). The computations are performed on a cluster of 128 quad core $2.0 \mathrm{GHz}$ processors. An exponential filter is applied along with the spectral method to eliminate the aliasing errors. The time integration is carried out by using the fourth-order Runge-Kutta method. We will describe the numerical method in detail in Sect. 2.

Several diagnostics are implemented to ensure that the numerical solutions are accurate. First, we compute the solutions of the linear equations,

$$
\theta_{t}+\kappa(-\Delta)^{\alpha} \theta=0
$$

and

$$
\theta_{t}+U \cdot \nabla \theta=0
$$

with a given velocity $U$, and compare with the exact solutions. Second, we perform careful resolution analysis to make sure any potential fronts are fully resolved. Third, we check if the computed solutions obey the two basic conservation laws, namely the conservation of the $L^{2}$-norm and of the helicity,

$$
\begin{aligned}
\frac{1}{2} \int \theta^{2}(x, t) d x & =\frac{1}{2} \int \theta_{0}^{2}(x) d x, \\
H(\theta) & =-\int \psi(x, t) \theta(x, t) d x=-\int \psi_{0}(x) \theta_{0}(x) d x .
\end{aligned}
$$

All these diagnostics indicate that the computations are accurate.

\section{Numerical Method}

We describe in this section the major numerical method used in the computations. Due to the periodic boundary condition, the pseudo-spectral scheme appears to be the most suitable method for the problem in hand (see, e.g. [34]). More precisely, we approximate the solution $\theta$ by $\tilde{\theta}$ of the form

$$
\tilde{\theta}(x, t)=\sum_{k_{1}, k_{2}=-N / 2}^{N / 2-1} \widehat{\theta}\left(k_{1}, k_{2}\right) e^{i k \cdot x}
$$

where $\widehat{\theta}$ denotes the Fourier transform of $\theta$, namely

$$
\widehat{\theta}\left(k_{1}, k_{2}\right)=\frac{1}{(2 \pi)^{2}} \int_{\mathbf{T}^{2}} \theta\left(x_{1}, x_{2}\right) e^{-i\left(k_{1} x_{1}+k_{2} x_{2}\right)} d x_{1} d x_{2}
$$


and $N$ is a fixed integer (usually $N=2^{m}$ for some positive integer $m$ ). It is easily seen from (1.1) that $\widehat{\theta}$ satisfies

$$
\partial_{t} \widehat{\theta}(k)=-i k_{1} \widehat{\left(u_{1} \theta\right)}(k)-i k_{2} \widehat{\left(u_{2} \theta\right)}(k)-2 \pi \kappa|k|^{2 \alpha} \widehat{\theta}(k),
$$

where $k=\left(k_{1}, k_{2}\right)$ is the wave number and $k_{1}, k_{2}=-N / 2, \ldots, N / 2-1$. The velocity field $u=\left(u_{1}, u_{2}\right)$ can be computed in the Fourier space by

$$
\widehat{u}(k)=i \frac{\left(-k_{2}, k_{1}\right)}{|k|} \widehat{\theta}(k) .
$$

The products $u_{1} \theta$ and $u_{2} \theta$ are computed in the physical space. With all these basic ingredients at our disposal, the evolution of the $N \times N$ modes of $\theta$, namely $\widehat{\theta}\left(j_{1}, j_{2}, t\right)$ for $j_{1}, j_{2}=-N / 2, \ldots, N / 2-1$ is then given by

$$
\partial_{t} \widehat{\Theta}=A \widehat{\Theta}
$$

where $\widehat{\Theta}$ is the matrix with the modes of $\theta$ being its entries and $A$ is a $N \times N$ matrix. This ordinary differential equation is then solved by the fourth-order Runge-Kutta method.

The serial pseudo-spectral algorithm described above is parallelized by slab decomposition for the purpose of parallel computations. Parallelized fast Fourier transform and inverse fast Fourier transform routines are used. More precisely, we use the MPI FFTW (Fast Fourier Transform in the West). The computations are done on total 128 machines with each of them featuring a Intel Pentium Xeon EM64T quad-core E5405 2.0 GHz processor in the Supercomputing Center, University of Oklahoma.

To reduce the aliasing errors that arise in our computations, we use an exponential filter. More precisely, we multiply each Fourier mode $\widehat{\theta}\left(k_{1}, k_{2}\right)$ by $f\left(2 k_{1} / N\right) f\left(2 k_{2} / N\right)$, where

$$
f(x)=\exp \left(-\alpha x^{m}\right) .
$$

Here $\alpha$ is usually taken to be $-\log \epsilon$ with $\epsilon$ being of the order of the machine precision and $m$ is an integer denoting the order of the filter. In our computations, we choose $\alpha=36$ and $m=19$. Figure 1 shows the graph of $f(x)$. When $m=19$, multiplying by $f$ keeps the $2 / 3$ modes unchanged and suppresses the $1 / 3$ higher frequency modes. This is a modification of the conventional way to cut off one-third of the high frequency modes, sometimes known as $2 / 3$ dealiasing rule. We shall briefly explain the motivation behind the application of a high order exponential filter instead of the traditional $2 / 3$ dealiasing rule. In the high order exponential filter method, we use a smoothing function of sufficiently high order which keeps a significant portion of the Fourier modes beyond the $2 / 3$ cut-off points. With the use of this method we have been able to significantly reduce the noises for high number of grid points. We learned the method of high order exponential filters from the paper of Thomas Hou and R. Li [37], in which the authors showed that the use of high order exponential filter captures about $12 \sim 15 \%$ more effective Fourier modes than the $2 / 3$ dealiasing method and produces a more accurate approximation of the solutions.

We briefly explain how this can remove the aliasing errors. For the sake of simple notation, we only consider the Fourier transform in one-direction. Let $x_{j}$ denote the grid points in that direction, namely

$$
x_{j}=\frac{2 \pi j}{N}, \quad j=0,1,2, \ldots, N-1 .
$$


Fig. 1 Exponential filter

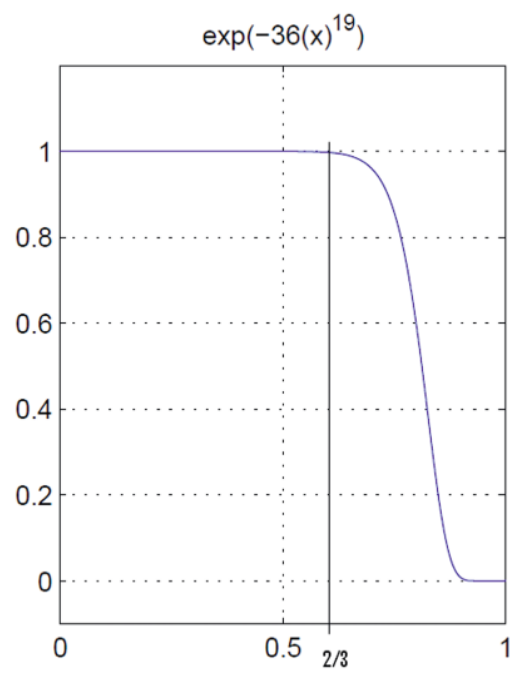

In the pseudo-spectral scheme, $\widehat{\theta}_{k}$ is computed through the discrete Fourier transform,

$$
\widehat{\theta_{k}}=\frac{1}{N} \sum_{j=0}^{N-1} \theta\left(x_{j}\right) e^{-i x_{j} k} .
$$

The SQG involves a quadratic nonlinearity and the truncated Fourier series for each factor in the nonlinear term are

$$
u_{j}=\sum_{m=-M / 2}^{M / 2-1} \widehat{u}_{m} e^{i m x_{j}}, \quad \theta_{j}=\sum_{n=-M / 2}^{M / 2-1} \widehat{\theta}_{n} e^{i n x_{j}}
$$

where we have filtered for $|m|>M / 2$ and $|n|>M / 2$. The discrete transform of the product $z_{j}=u_{j} \theta_{j}$ is

$$
\widehat{z}_{k}=\sum_{j} z_{j} e^{-i k x_{j}}
$$

Inserting (2.2) in (2.3) yields

$$
\widehat{z}_{k}=\sum_{m+n=k} \widehat{u}_{m} \widehat{\theta}_{n}+\sum_{m+n=k \pm N} \widehat{u}_{m} \widehat{\theta}_{n}
$$

The second term on the right side is the aliasing error. If we filter so that $\widehat{u}_{m}=0$ and $\widehat{\theta}_{n}=0$ for $|m|>M / 2$ and $|n|>M / 2$ in the above formula and want $\widehat{z}_{k}=0$ for all $|k|>M / 2$, then the largest value of $M$ required to make the aliasing term zero happens when $m=m=$ $-M / 2$ and $k=M / 2-1$, namely

$$
-\frac{M}{2}-\frac{M}{2} \leq \frac{M}{2}-1-N \quad \text { or } \quad M \geq \frac{2}{3}(N+1) .
$$


Table 1 Error for Inviscid SQG equations

\begin{tabular}{lllll}
\hline$t$ & 1 & 5 & 10 & 15 \\
\hline Max error & $1.1507 \mathrm{e}-004$ & $1.7550 \mathrm{e}-004$ & $1.8507 \mathrm{e}-004$ & $1.9507 \mathrm{e}-004$ \\
Relative error & $0.11 \%$ & $0.11 \%$ & $0.11 \%$ & $0.12 \%$ \\
\hline
\end{tabular}

Table 2 Error for dissipative SQG equations

\begin{tabular}{lllll}
\hline$t$ & 1 & 5 & 10 & 15 \\
\hline Max error & $1.302 \mathrm{e}-003$ & $1.402 \mathrm{e}-003$ & $1.422 \mathrm{e}-003$ & $1.501 \mathrm{e}-003$ \\
Relative error & $0.41 \%$ & $0.41 \%$ & $0.41 \%$ & $0.42 \%$ \\
\hline
\end{tabular}

We now test the accuracy of the numerical scheme on two linear equations. The first linear equation assumes the form

$$
\theta_{t}+U \cdot \nabla \theta=0, \quad \theta(x, 0)=\theta_{0}(x)
$$

with $U=\left(U_{1}, U_{2}\right)$ being a constant velocity. This transport equation admits the explicit solution

$$
\theta\left(x_{1}, x_{2}, t\right)=\theta_{0}\left(x_{1}-U_{1} t, x_{2}-U_{2} t\right) .
$$

The numerical method is implemented and the solution of (2.4) is computed with $U_{1}=1$ and $U_{2}=1$. Table 1 lists the errors between the exact solution and the computed solution at various times.

The second equation we have tested is the linear dissipative equation

$$
\theta_{t}+\kappa(-\Delta)^{\alpha} \theta=0
$$

with $\kappa=0.001$ and $\alpha=0.4$. The explicit solution $\theta$ satisfies

$$
\hat{\theta}\left(k_{1}, k_{2}, t\right)=\hat{\theta}_{0}\left(k_{1}, k_{2}\right) e^{-\kappa\left(k_{1}^{2}+k_{2}^{2}\right)^{\alpha} t} .
$$

The following lists the absolute and relative errors at several times. Table 2 represents the absolute and relative errors at several times. The tests indicate that the numerical algorithm, when implemented on these linear equations, is extremely accurate.

\section{Numerical Results}

Our major numerical results are presented here in two subsections. The first subsection contains the results for the inviscid SQG equation while the second is devoted to the dissipative SQG equation.

\subsection{The inviscid SQG equation}

In this subsection, solutions of the inviscid SQG equation emanating from the data given in (1.4), (1.5) and (1.6) are computed. We investigate if these solutions can develop any finitetime singularity and examine their large-time behavior. For these purposes, the level curves 
Fig. 2 Contours of $\theta$ for data in (1.4), $t=0,2,4,6$
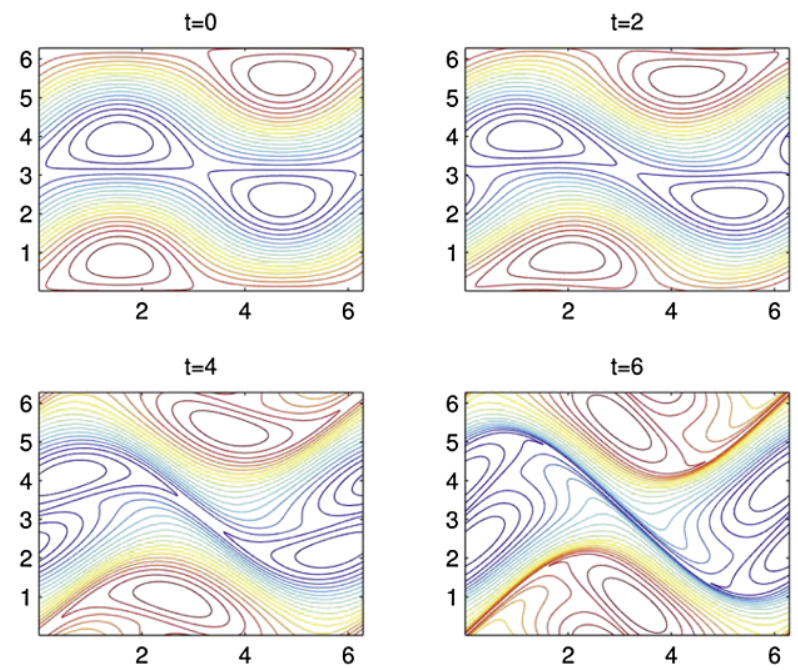

of each solution $\theta$ at various times are plotted. Special attention is paid to the numerical solutions corresponding to the data in (1.4), which exhibit the most singular behavior. We record the evolution of the spatial regions in which $\left|\nabla \theta\left(x_{1}, x_{2}, t\right)\right| \geq c\|\nabla \theta(\cdot, t)\|_{L^{\infty}}$ and carry out a detailed resolution analysis. In addition, we closely monitor two key conserved quantities, the $L^{2}$-norm of $\theta$ and the helicity.

The first set of numerical experiments is done for the initial data given in (1.4), namely

$$
\theta(x, 0)=\sin \left(x_{1}\right) \sin \left(x_{2}\right)+\cos \left(x_{2}\right) .
$$

Figure 2 shows the evolution of contours of $\theta$ from $t=0$ to $t=6$. Figure 3 features an enlarged plot of the contour at $t=6$, while Fig. 4 provides the surface plot of $\theta$ at $t=7$.

The numerical results obtained here for up to $t=7$ are very close to those that have previously been obtained in $[15,52]$ and [16]. As indicated in [15], $\|\nabla \theta(\cdot, t)\|_{\infty}$ grows rather rapidly around $t=7$. To determine if a potential singularity is possible near $t=7$, we carefully monitored the behavior of $\theta$ in the time interval [7, 8.5]. We did not notice any drastic change in the behavior of the solution. Figures 5 and 6 show the plots of $\theta$ at $t=7.5$ and $t=8$, respectively. As the time evolves, the maximum gradient $\|\nabla \theta(\cdot, t)\|_{\infty}$ continues to grow. It appears that a sharp front is developing along the hyperbolic saddle. However, as our computations proceed, $\|\nabla \theta(\cdot, t)\|_{\infty}$ stops increasing at around $t=13.5$. Figures 7 and 8 present the contour plots of $\theta$ for $t=8$ to $t=14$, and for $t=16$ and $t=20$. To further understand the behavior of $\nabla \theta$, we traced the region of $\left(x_{1}, x_{2}\right)$ for which

$$
\left|\nabla \theta\left(x_{1}, x_{2}, t\right)\right| \geq 0.8\|\nabla \theta(\cdot, t)\|_{L^{\infty}} .
$$

Figure 9 presents its evolution for $t=8$ to $t=14$. These plots indicate that the potential sharp front of $\theta$ does not intensify itself at a fixed location. Figures 10, 11 and 12 plot $\|\nabla \theta(\cdot, t)\|_{\infty}$ versus $t$ for three different resolutions, $512^{2}, 1024^{2}$ and $2048^{2}$. They clearly show that the maximal gradient decreases as the time evolves after $t=13.5$. The reason for the decay is not clear, but we plotted the contour curves of $\theta$ at $t=13.5$ and marked where the values of $\left|\nabla^{\perp} \theta\right|$ are large (see Figs. 13 and 14). We have performed careful resolution analysis to ensure that any potential sharp front is fully resolved. As an example, we present 


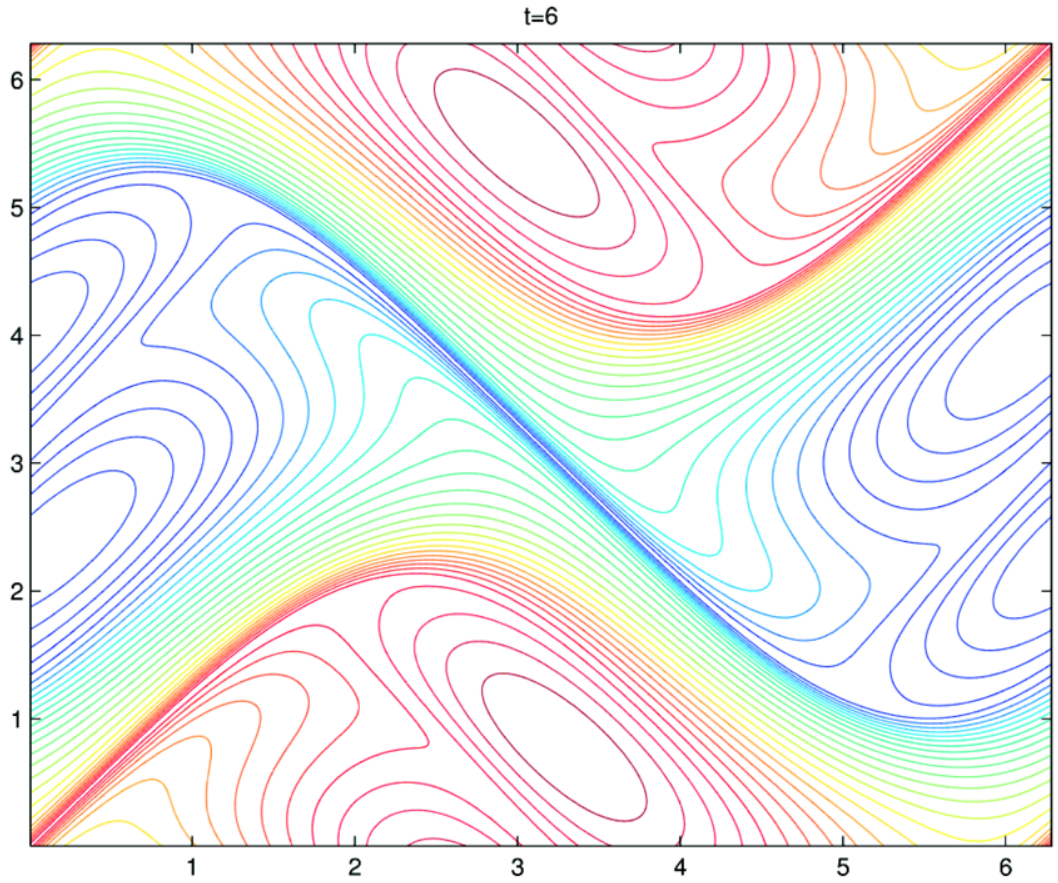

Fig. 3 Enlarged plot of the contours of $\theta, t=6$

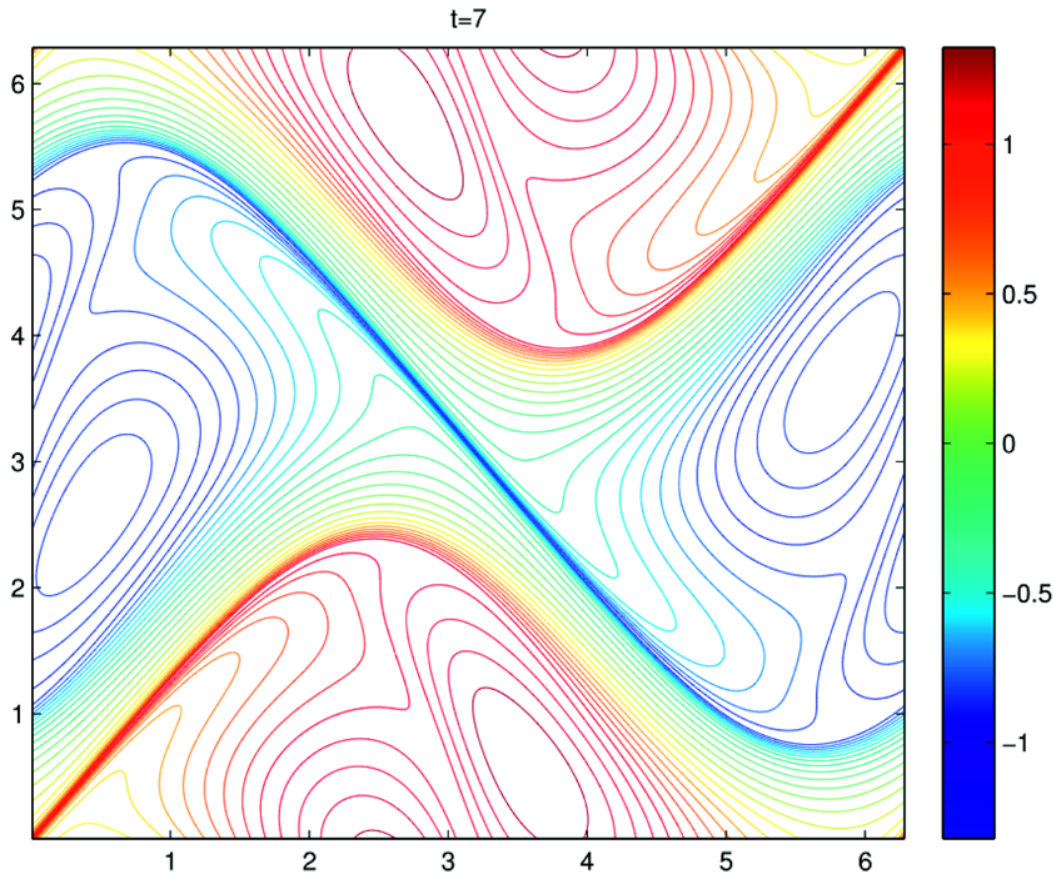

Fig. 4 Enlarged plot of the contours of $\theta, t=7$ 


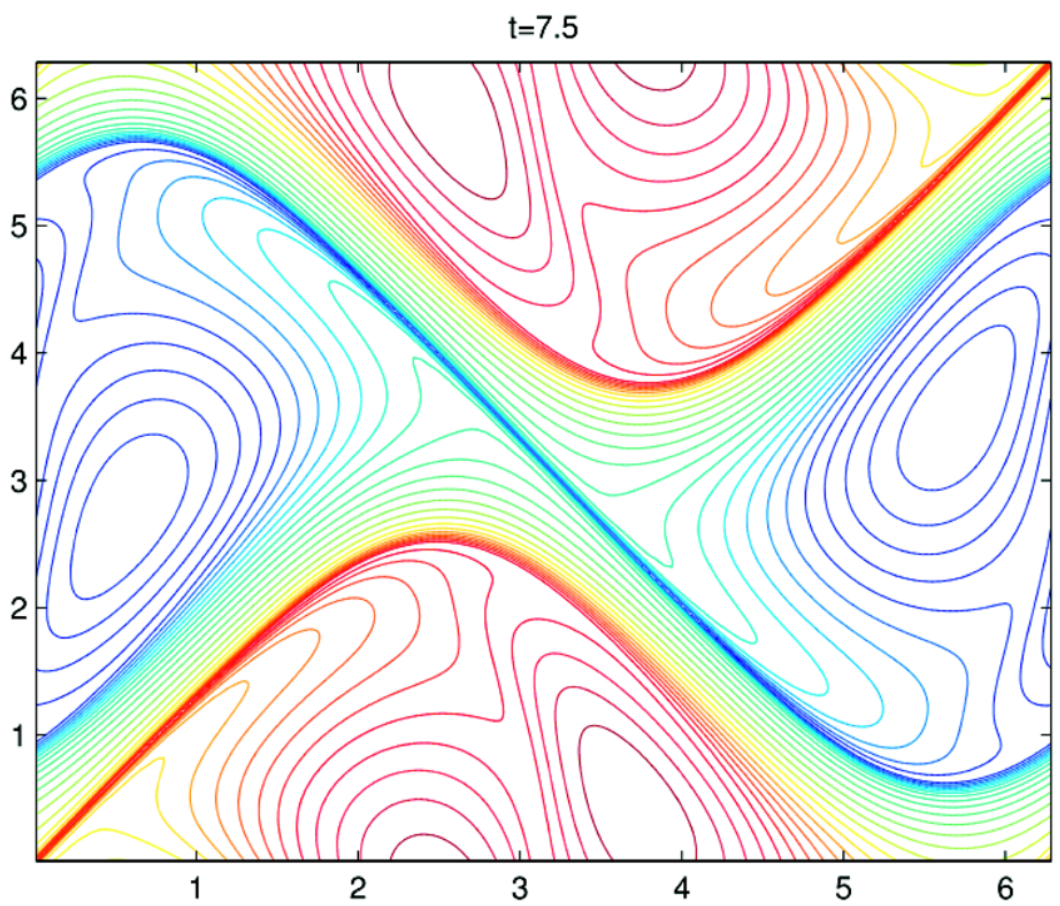

Fig. 5 Enlarged plot of the contours of $\theta, t=7.5$

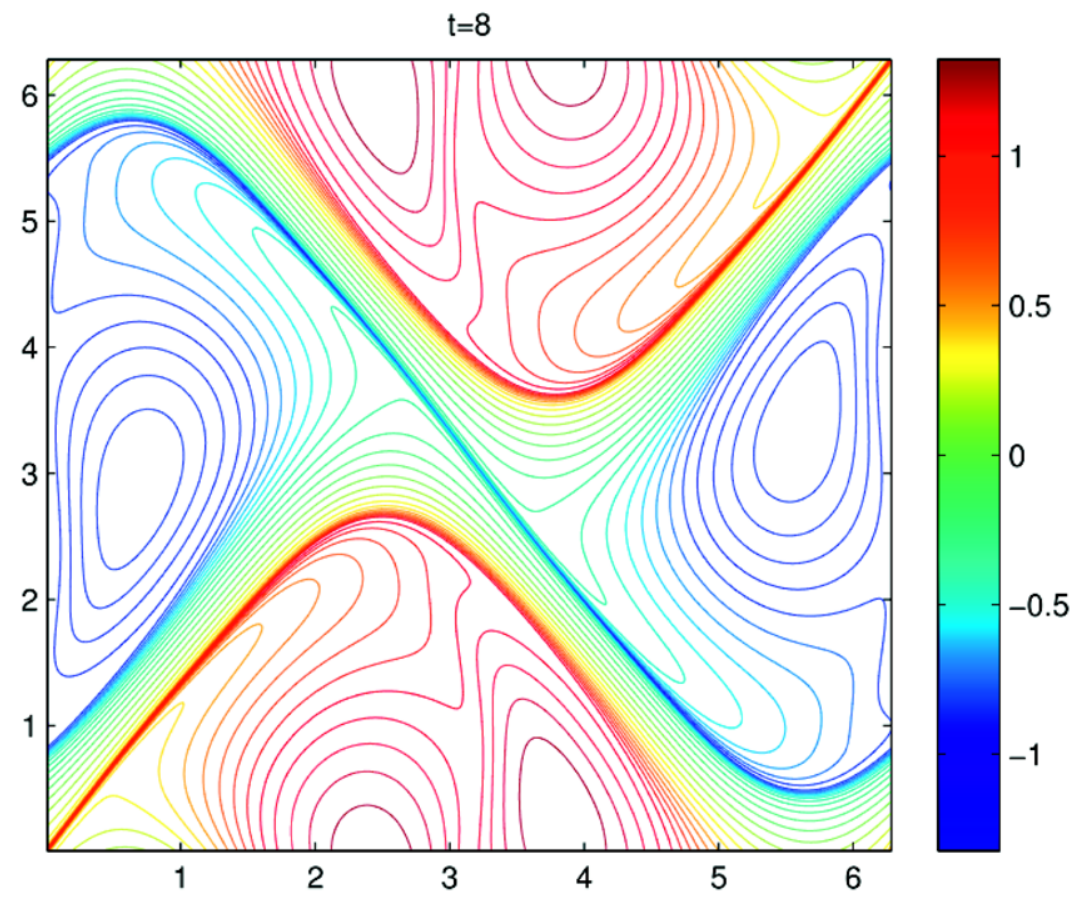

Fig. 6 Enlarged plot of the contours of $\theta, t=8$ 

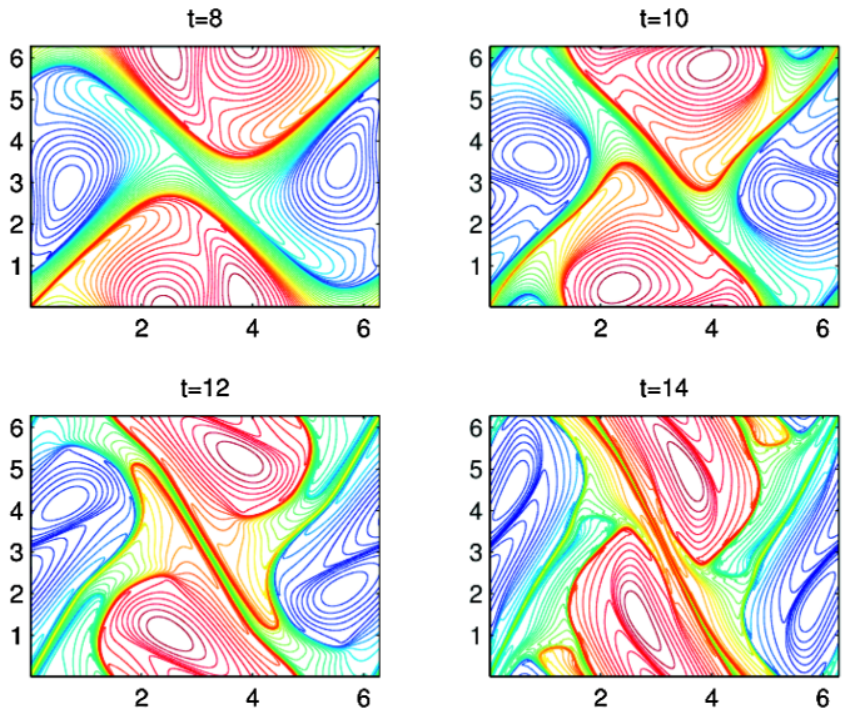

Fig. 7 Contours of $\theta$ for data in (1.4), $t=8,10,12,14$
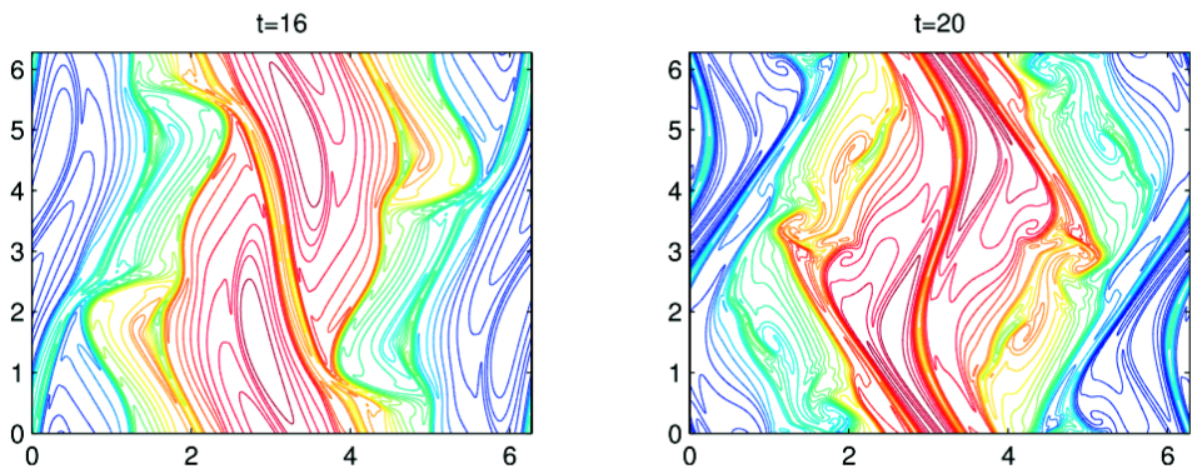

Fig. 8 Contours of $\theta$ for data in (1.4), $t=16,20$

the contour plots of $\theta$ at $t=16$ with various resolutions: $N=256,512,1024,2048$ and 4096. The plots for $N=256,512,1024$ and 2048 are given in Figs. 15, 16, 17 and 18, respectively. When $N=4096$ points are used in each direction, the data for the contour plot of $\theta$ is very big and we split the plot into four parts with each on one of the quarters: $[0, \pi] \times[0, \pi],[0, \pi] \times[\pi, 2 \pi],[\pi, 2 \pi] \times[0, \pi]$ and $[\pi, 2 \pi] \times[\pi, 2 \pi]$ (see Fig. 19).

We carefully monitored two key conserved quantities throughout the calculations, the $L^{2}$ norm of $\theta$ and the helicity. Tables 3 and 4 record the numerical values of these quantities at various times. In the tables KE denotes the $L^{2}$-norm and $H(\theta)$ denotes the helicity, namely

$$
\mathrm{KE}^{2}=\frac{1}{2} \int_{\mathbf{T}^{2}} \theta^{2}(x, t) d x, \quad H(\theta)=-\int_{\mathbf{T}^{2}} \psi(x, t) \theta(x, t) d x .
$$

The second set of numerical experiments computes the solution of the inviscid SQG equations with the initial data given in (1.5). Figures 20, 21 and 22 plot the contours of $\theta$ 

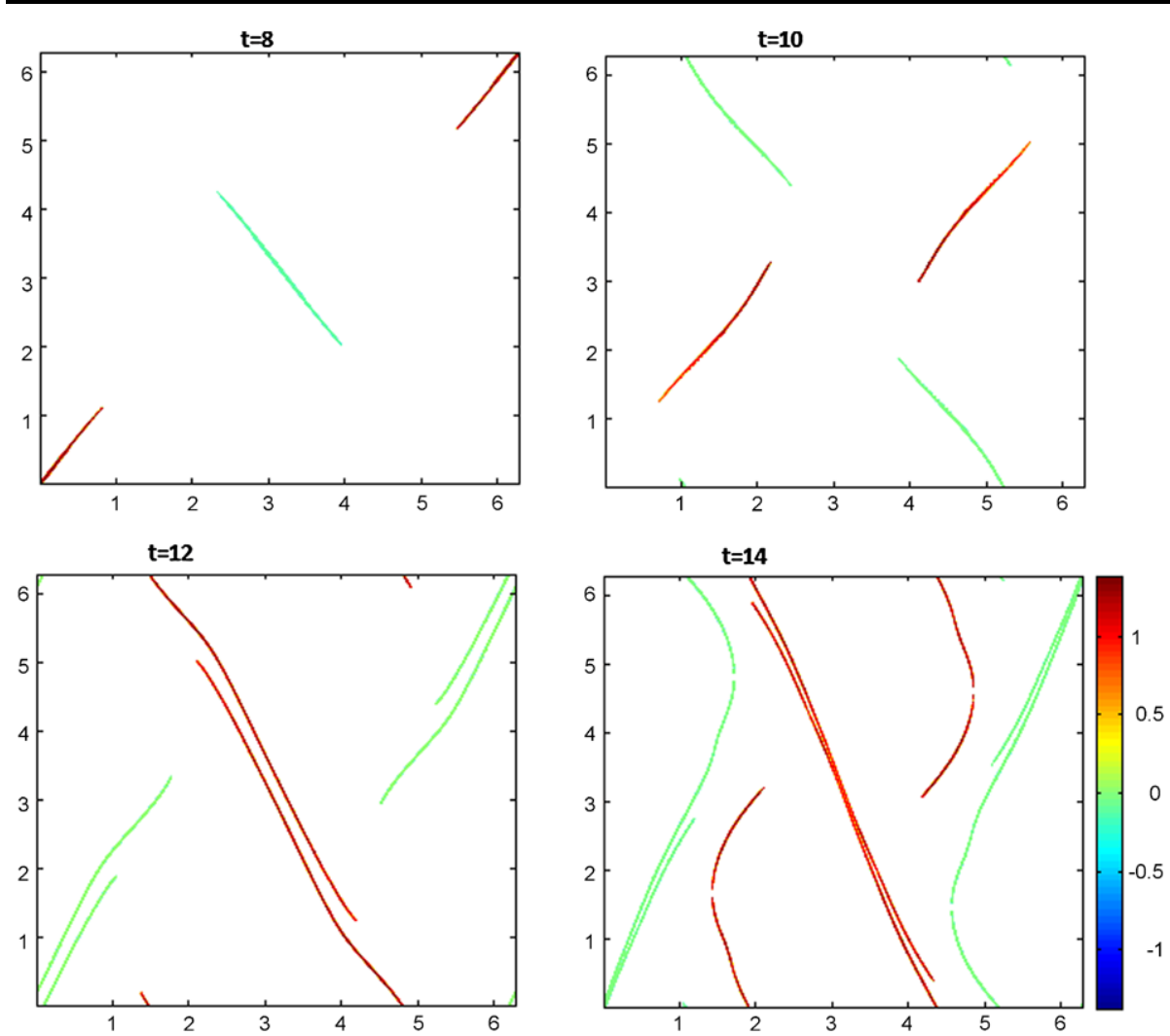

Fig. 9 Regions in which $\left|\nabla \theta\left(x_{1}, x_{2}, t\right)\right| \geq 0.8\|\nabla \theta(\cdot, t)\|_{L^{\infty}}$

Fig. $10\|\nabla \theta(\cdot, t)\|_{\infty}$ versus $t$ (for data in (1.4)) for $N=512$

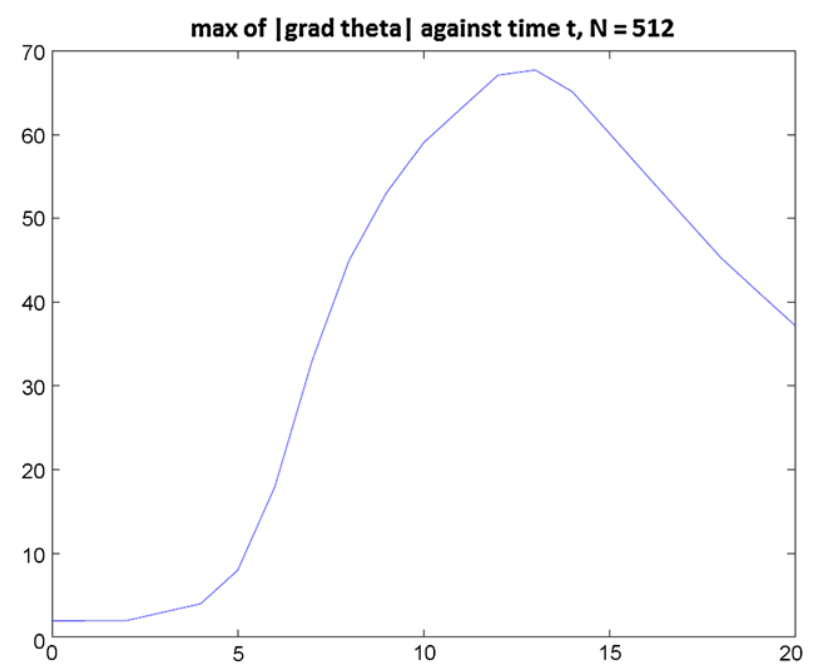


Fig. $11\|\nabla \theta(\cdot, t)\|_{\infty}$ versus $t$ (for data in (1.4)) for $N=1024$

Fig. $12\|\nabla \theta(\cdot, t)\|_{\infty}$ versus $t$ (for data in (1.4)) for $N=2048$
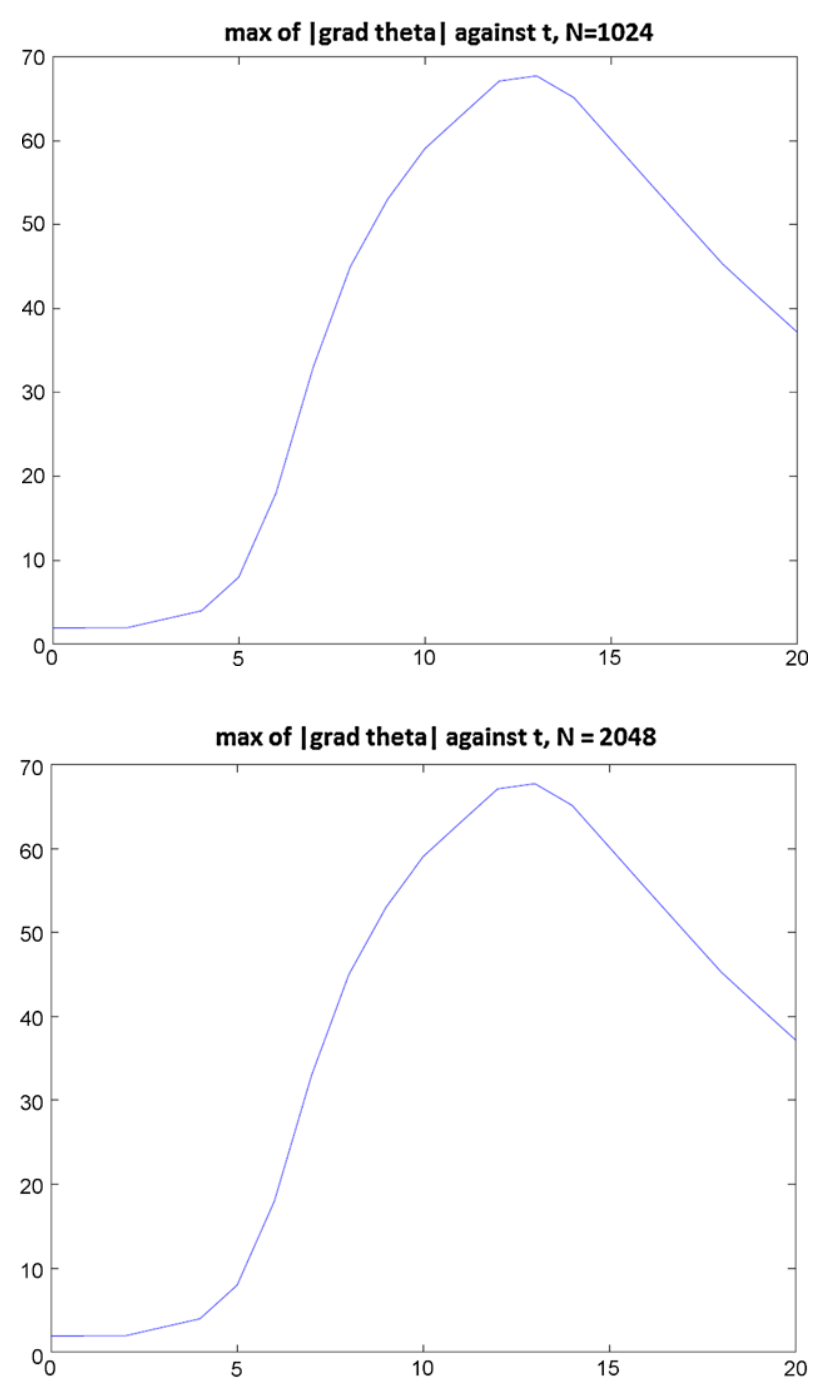

from $t=0$ to $t=6$, from $t=7$ to $t=10$ and from $t=11$ to $t=14$, respectively. Figures 23 and 24 provide the enlarged plots at $t=6$ and $t=8$. The numerical solution does not exhibit any finite time singular behavior.

The third set of numerical experiments is done for the solution of the inviscid SQG equation with the initial data given in (1.6). Figures 25 and 26 present the evolution of the contours of $\theta$ from $t=0$ to $t=6$ and from $t=8$ to $t=14$, respectively. Figures 27 and 28 show the enlarged plots at $t=7$ and $t=8$. No singular behavior has been detected in the calculations.

\subsection{The Dissipative SQG Equation}

This subsection presents numerical results for the dissipative SQG equation,

$$
\theta_{t}+u \cdot \nabla \theta+\kappa(-\Delta)^{\alpha} \theta=0
$$




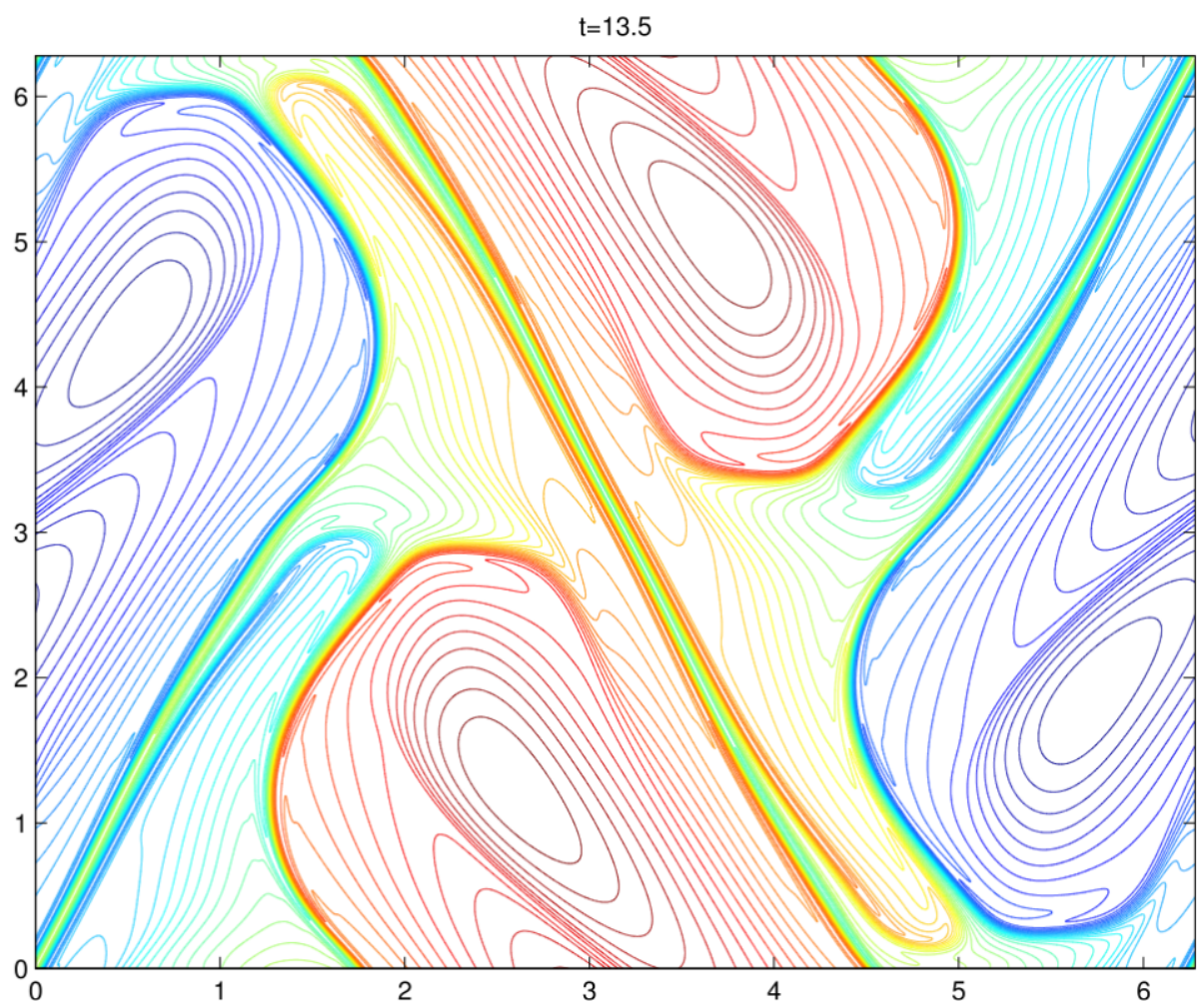

Fig. 13 Contours of $\theta$ for data in (1.4), $t=13.5$

Fig. 14 Spatial regions with $|\nabla \theta| \geq 0.8 \max |\nabla \theta|$ at $t=13.5$

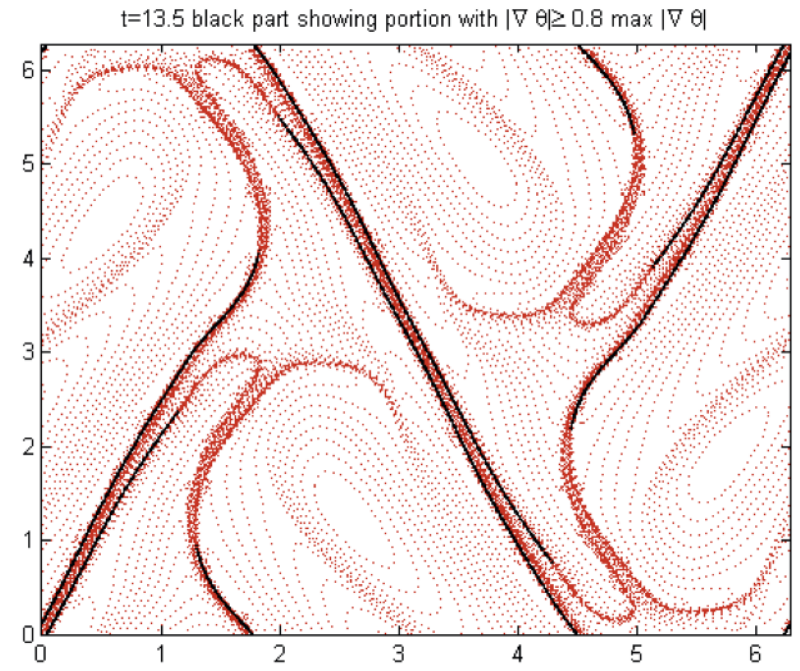




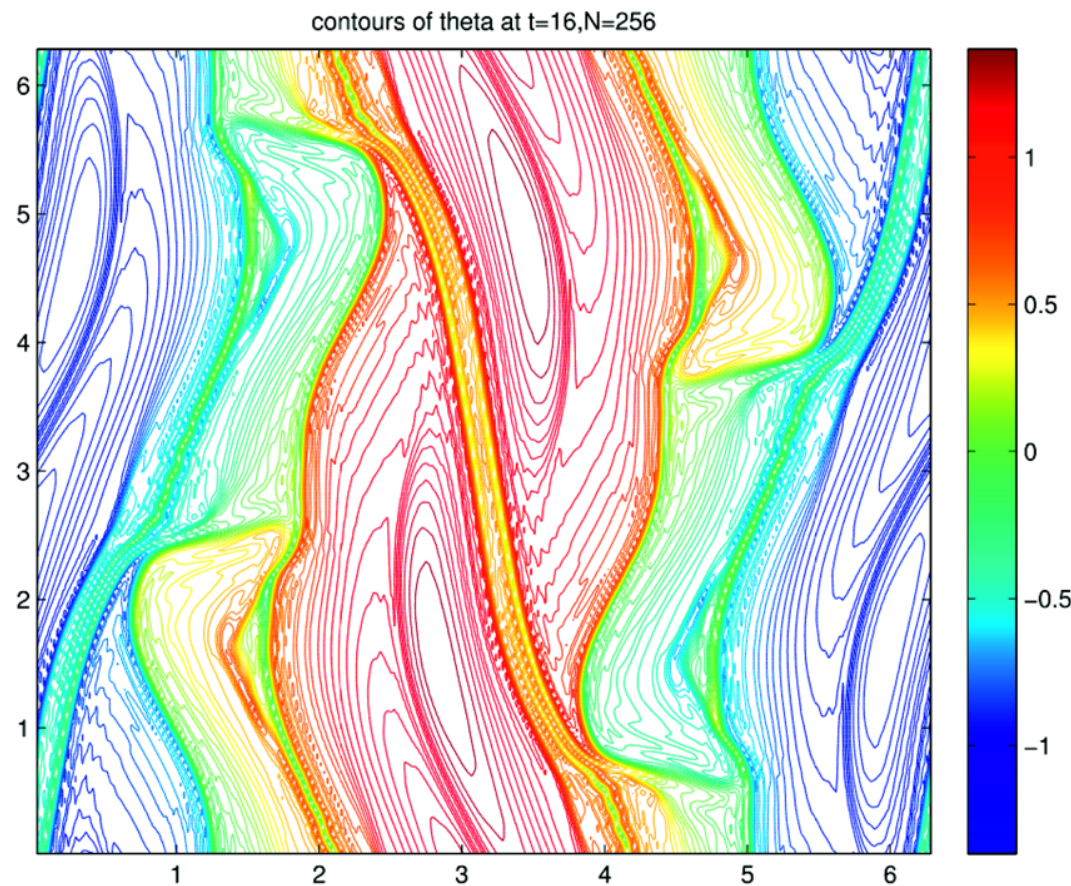

Fig. 15 Contours of $\theta$ for data in (1.4), $t=16, N=256$

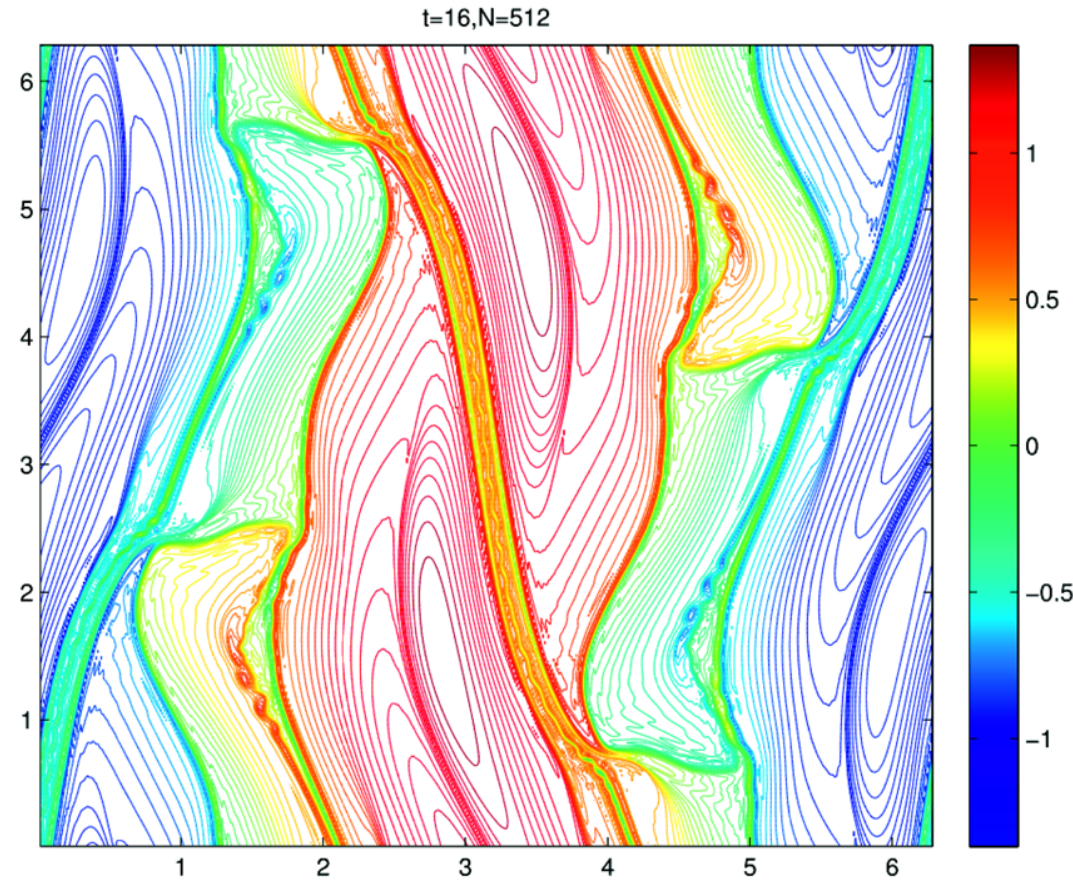

Fig. 16 Contours of $\theta$ for data in (1.4), $t=16, N=512$ 


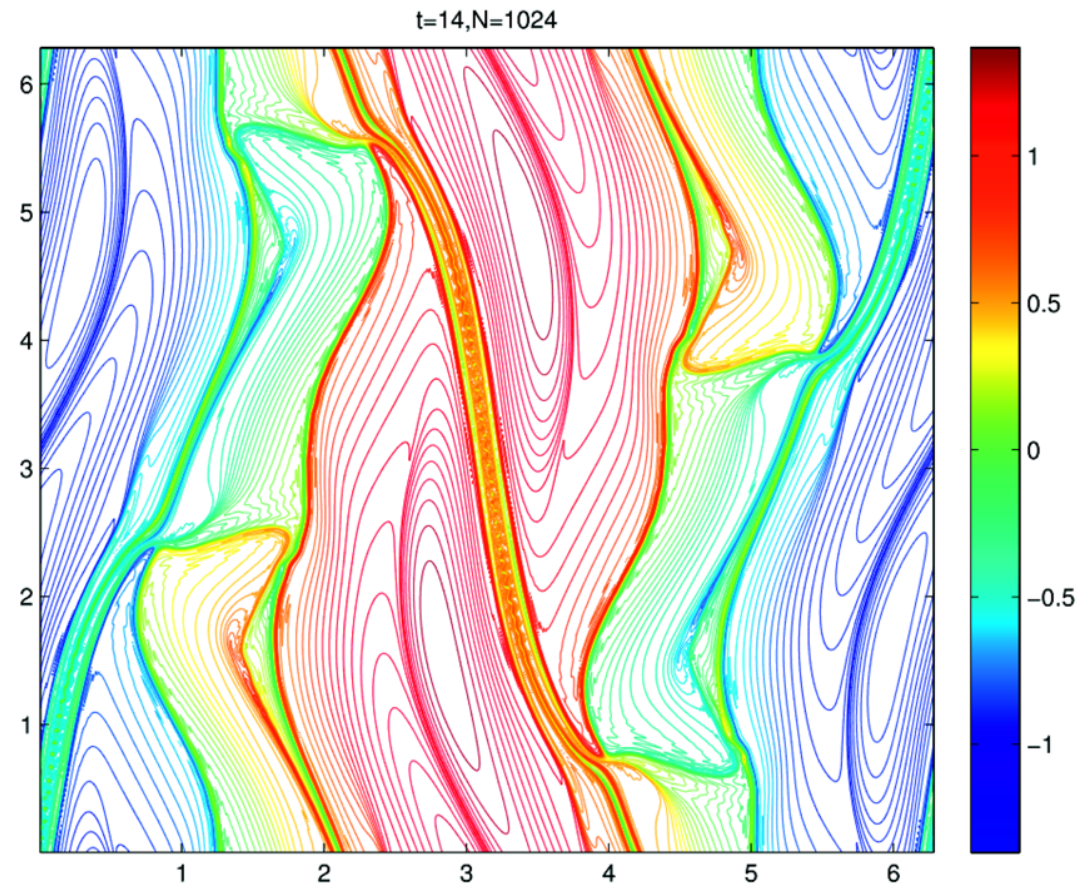

Fig. 17 Contours of $\theta$ for data in (1.4), $t=16, N=1024$

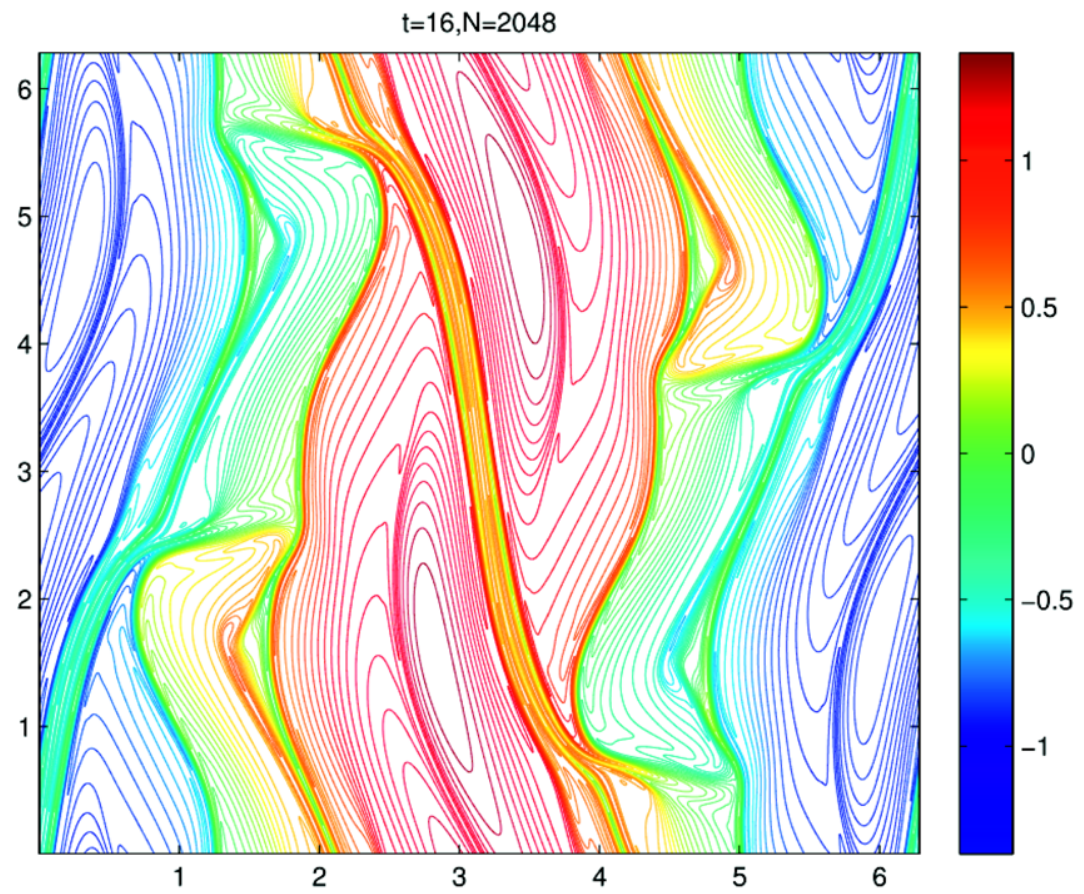

Fig. 18 Contours of $\theta$ for data in (1.4), $t=16, N=2048$ 

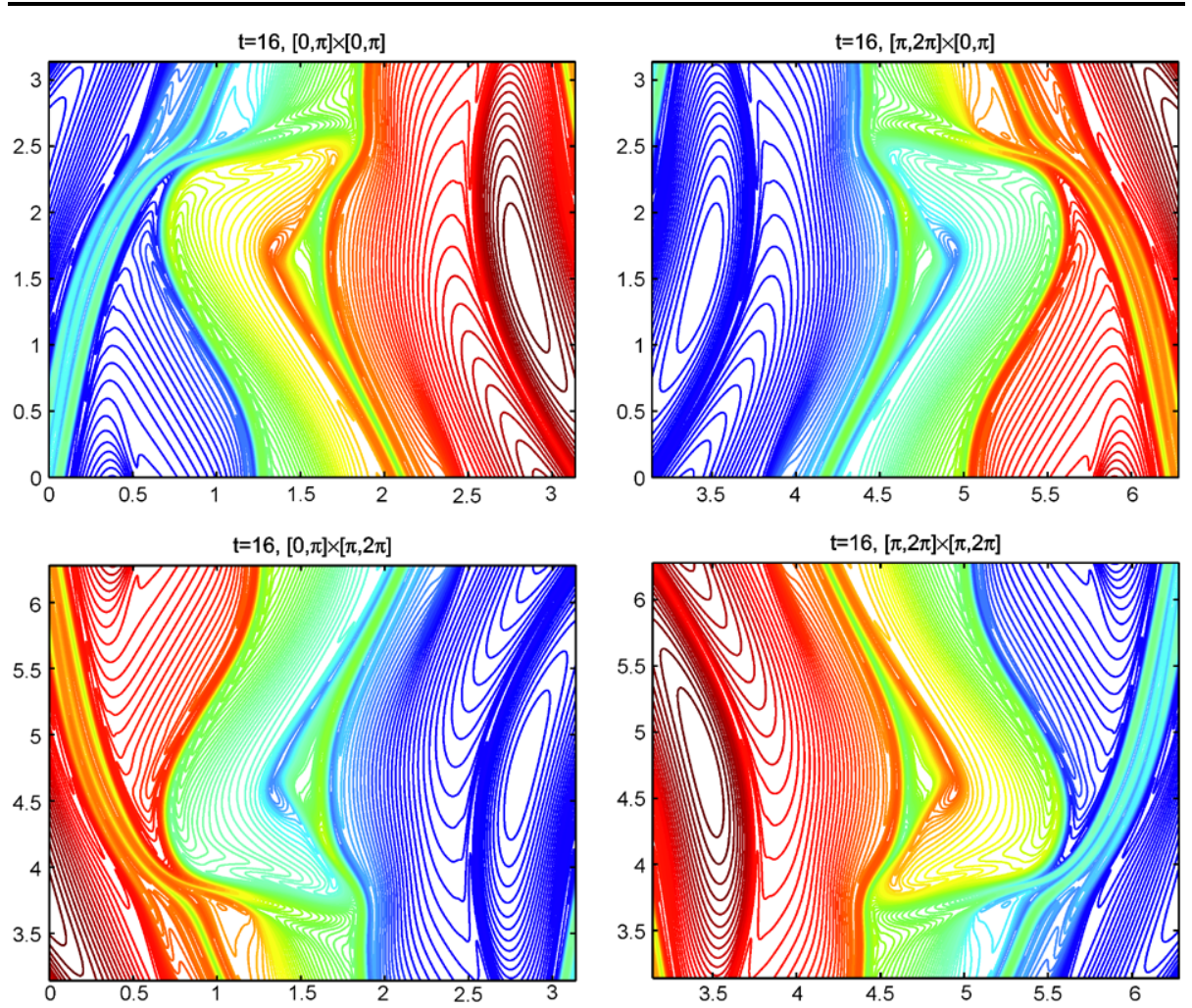

Fig. 19 Contours of $\theta$ for data in (1.4), $t=16, N=4096$

Table 3 Kinetic Energy for initial data in (1.4)

\begin{tabular}{llllll}
\hline$t$ & 0 & 1 & 2 & 4 & 6 \\
\hline $\mathrm{KE}$ & 14.804406 & 14.804406 & 14.804407 & 14.804407 & 14.804409 \\
\hline$t$ & 8 & 10 & 12 & 14 & 16 \\
\hline $\mathrm{KE}$ & 14.80441 & 14.80441 & 14.80443 & 14.80443 & 14.80443 \\
\hline
\end{tabular}

Table 4 Helicity for data in (1.4)

\begin{tabular}{llllll}
\hline$t$ & 0 & 1 & 2 & 4 & 6 \\
\hline $\mathrm{H}(\theta)$ & 4.2523 & 4.2523 & 4.2523 & 4.2524 & 4.2524 \\
\hline$t$ & 8 & 10 & 12 & 14 & 16 \\
\hline $\mathrm{H}(\theta)$ & 4.2524 & 4.2525 & 4.2523 & 4.2523 & 4.2523 \\
\hline
\end{tabular}

The aim of the numerical experiments described here is two-fold: first, to see if the solutions of the supercritical SQG equation can develop any finite-time singularity; and second, to determine if $\alpha=\frac{1}{2}$ is critical and how $\kappa$ affects the regularity of solutions. Attention will be focused on the solution corresponding to the initial data given in (1.4). 

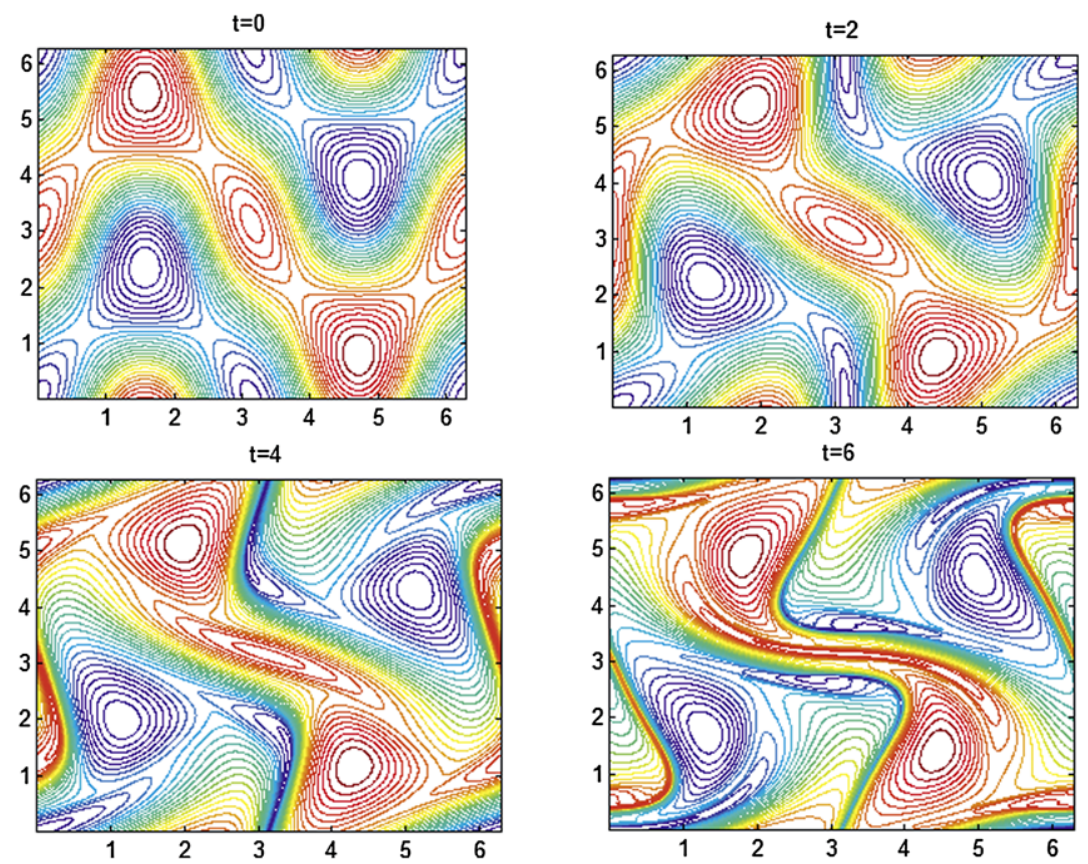

Fig. 20 Contours of $\theta$ for data in (1.5), $t=0,2,4,6$
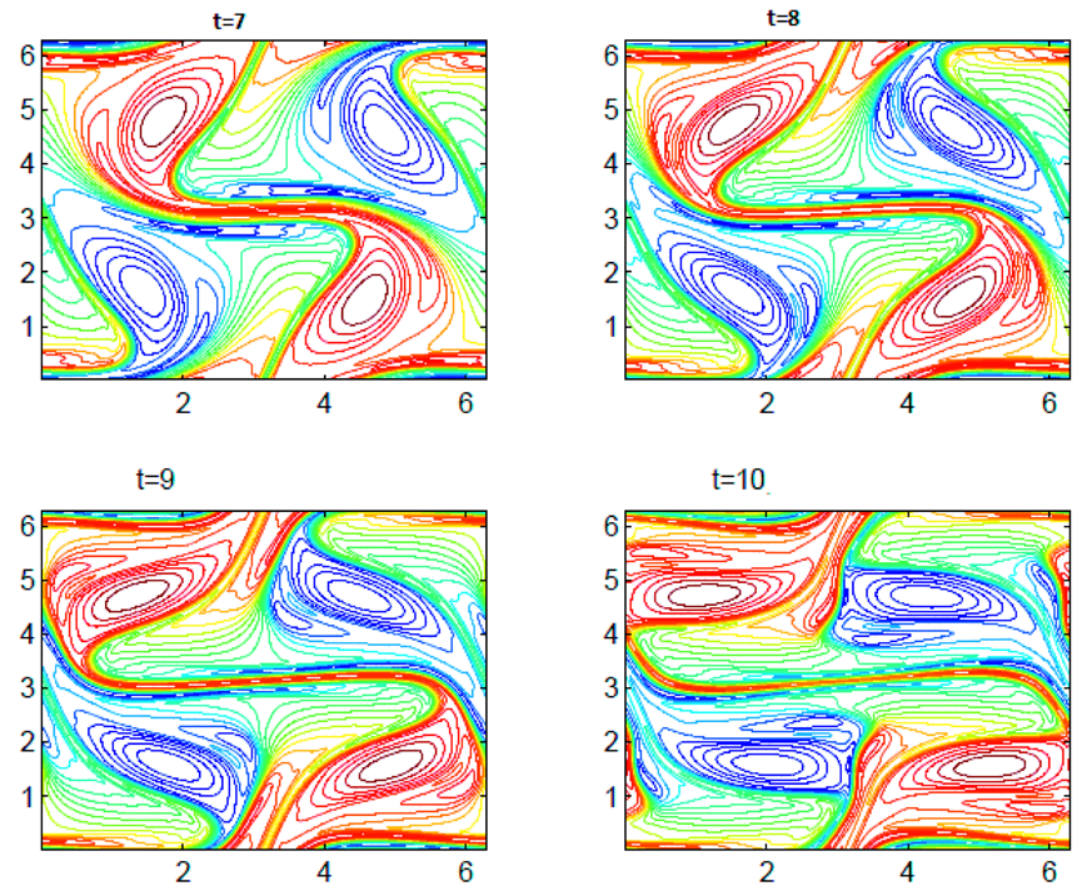

Fig. 21 Contours of $\theta$ for data in (1.5), $t=7,8,9,10$ 

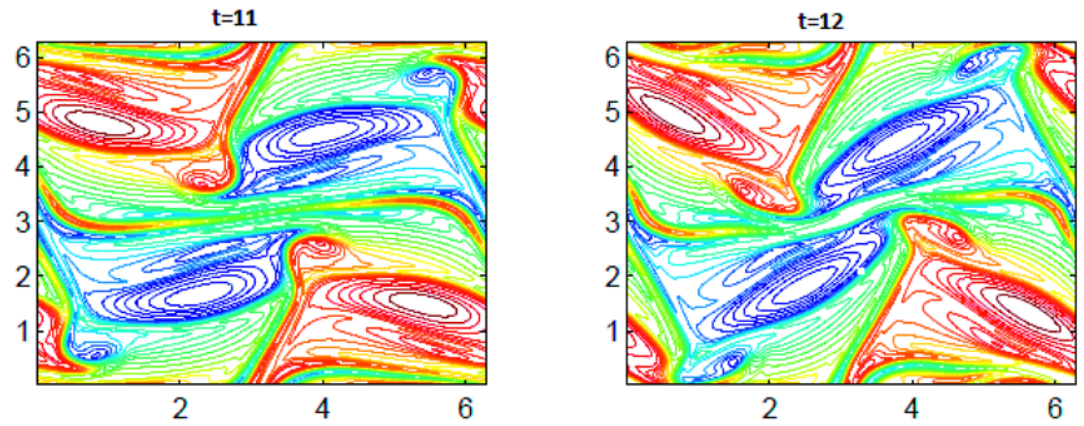

$\mathrm{t}=13$
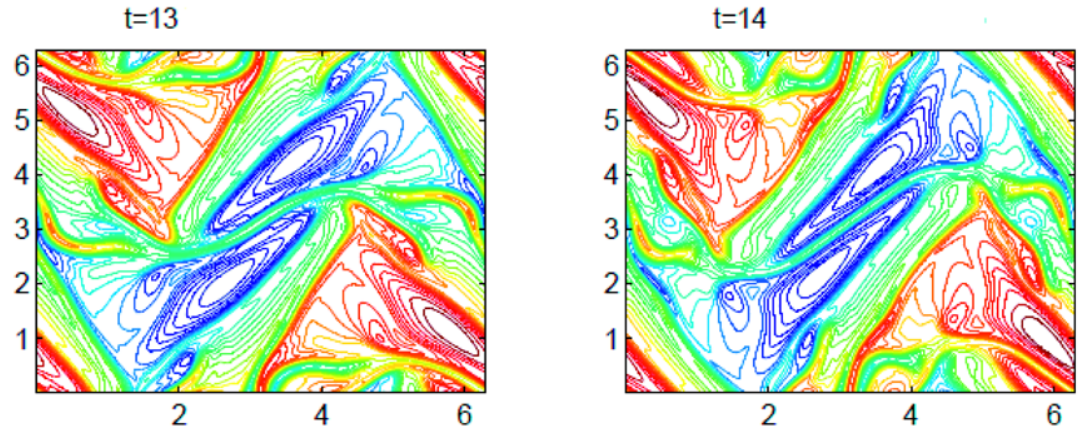

Fig. 22 Contours of $\theta$ for data in (1.5), $t=11,12,13,14$

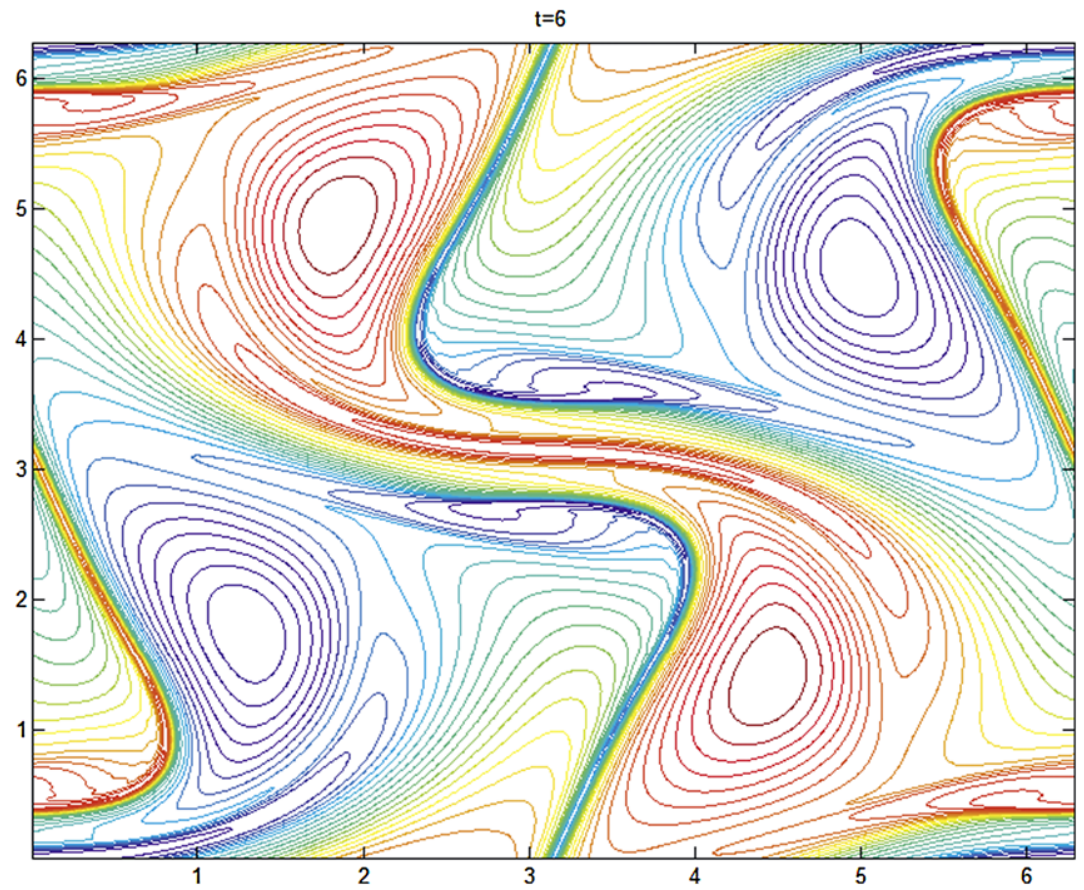

Fig. 23 Enlarged contours of $\theta$ for data in (1.5), $t=6$ 


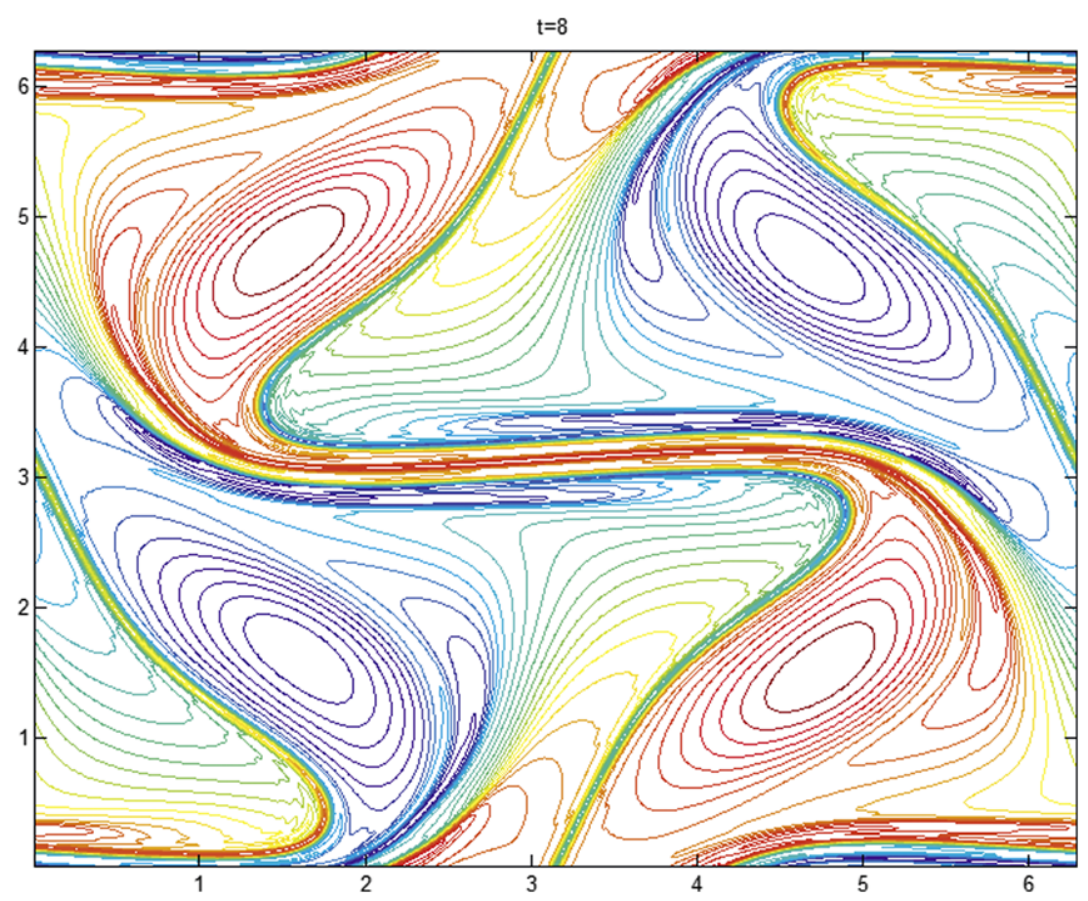

Fig. 24 Contours of $\theta$ for data in (1.5), $t=8$
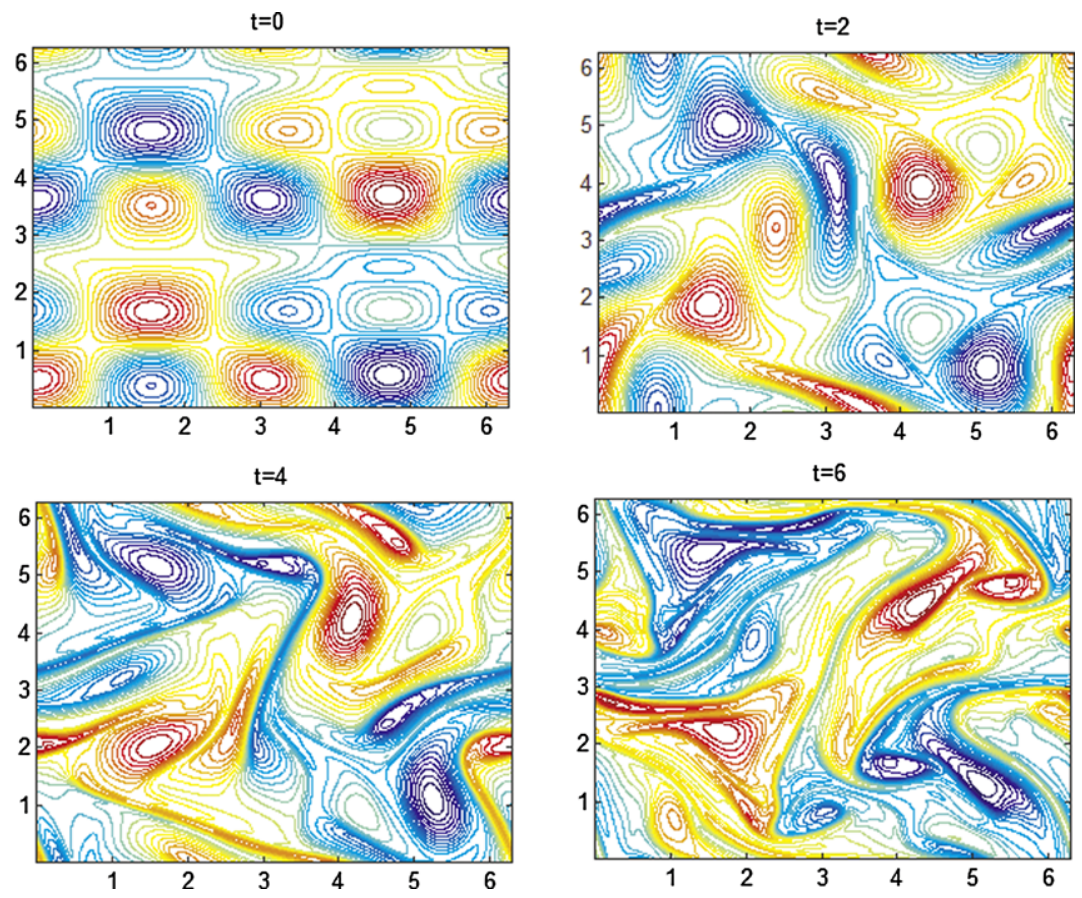

Fig. 25 Contours of $\theta$ for data in (1.6), $t$ from 0 to 6 

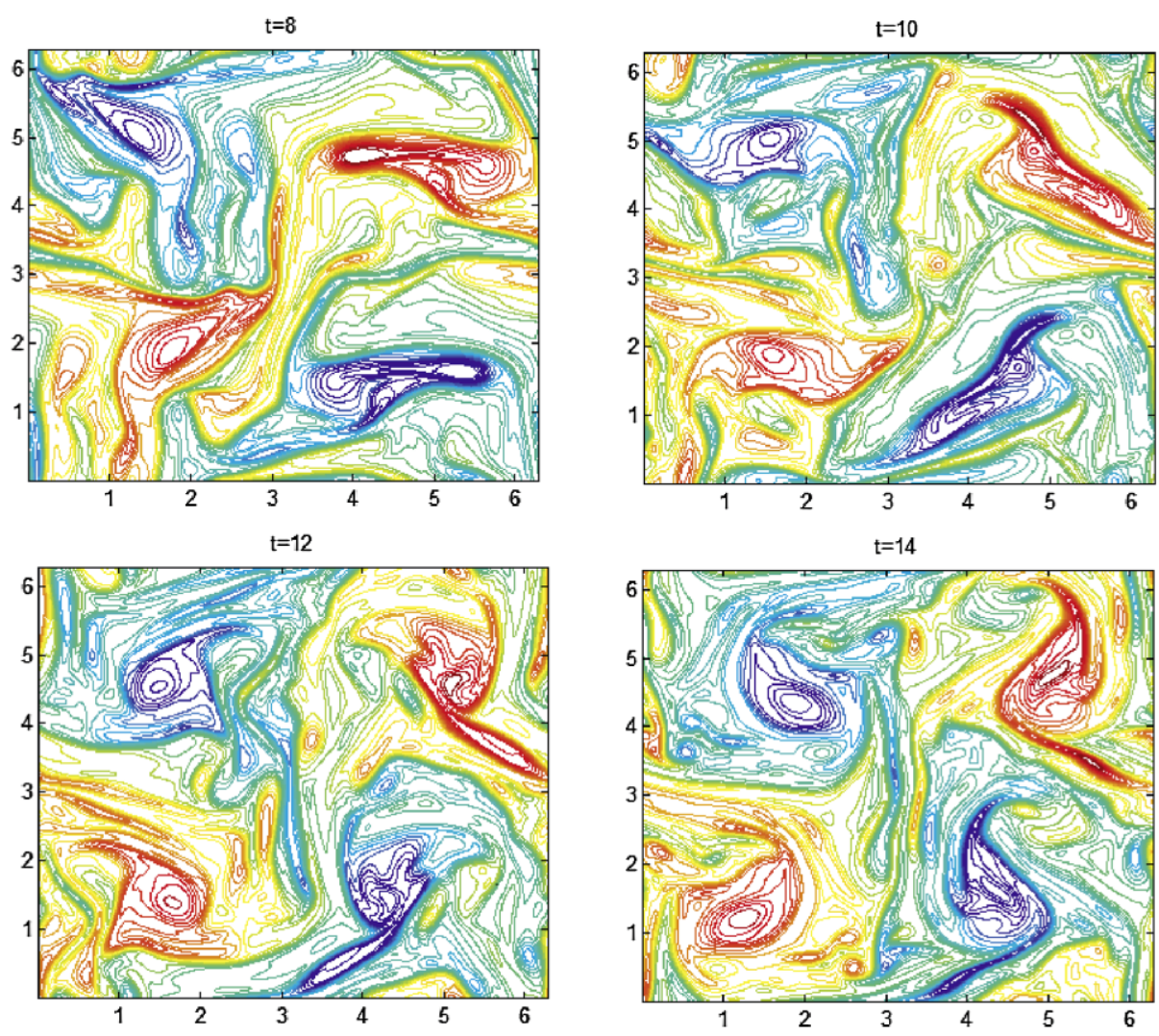

Fig. 26 Contours of $\theta$ for data in (1.6), $t$ from 8 to 14

As mentioned in the introduction, $\alpha=\frac{1}{2}$ appears to be critical in the theoretical study of the global regularity issue. When $\alpha>\frac{1}{2}$, the dissipation is sufficient to control the nonlinearity and (1.1) has a global classical solution for any sufficiently smooth initial data. When $\alpha=\frac{1}{2}$, the dissipation can barely bound the nonlinearity and the energy type estimates no longer yield global (in time) bounds. If $\kappa$ is sufficiently large compared to the initial data, the global regularity still hold, as shown in by Constantin, Córdoba and Wu [13]. The critical case with a general large data was recently completely resolved [4, 41]. The global regularity issue for the supercritical case $\alpha<\frac{1}{2}$ is in general open, but the results in [18, 19], and [31] imply that any potential finite-time singularity must occur in the regularity window between $L^{\infty}$ and $C^{1-2 \alpha}$.

The first round of computations is done for the supercritical case $\alpha=0.4$ and $\kappa=$ 0.001 . The solution is computed at various resolutions and no finite-time singularity is detected. Figure 29 depicts the evolution of the contours of $\theta$ from $t=6$ to $t=20$ with $N=2048$.

The second set of experiments computes the solutions with $\kappa=0.001$ and $\alpha$ varying from 0.45 to 0.51 . The intention is to test the criticality of $\alpha=0.5$. The contours at $t=$ 14 for $\alpha=0.45,0.48,0.49,0.5,0.51$ are plotted in Fig. 30. The fact that these contours tend out to be very similar suggests that $\alpha=0.5$ may not be special for this numerical solution. 


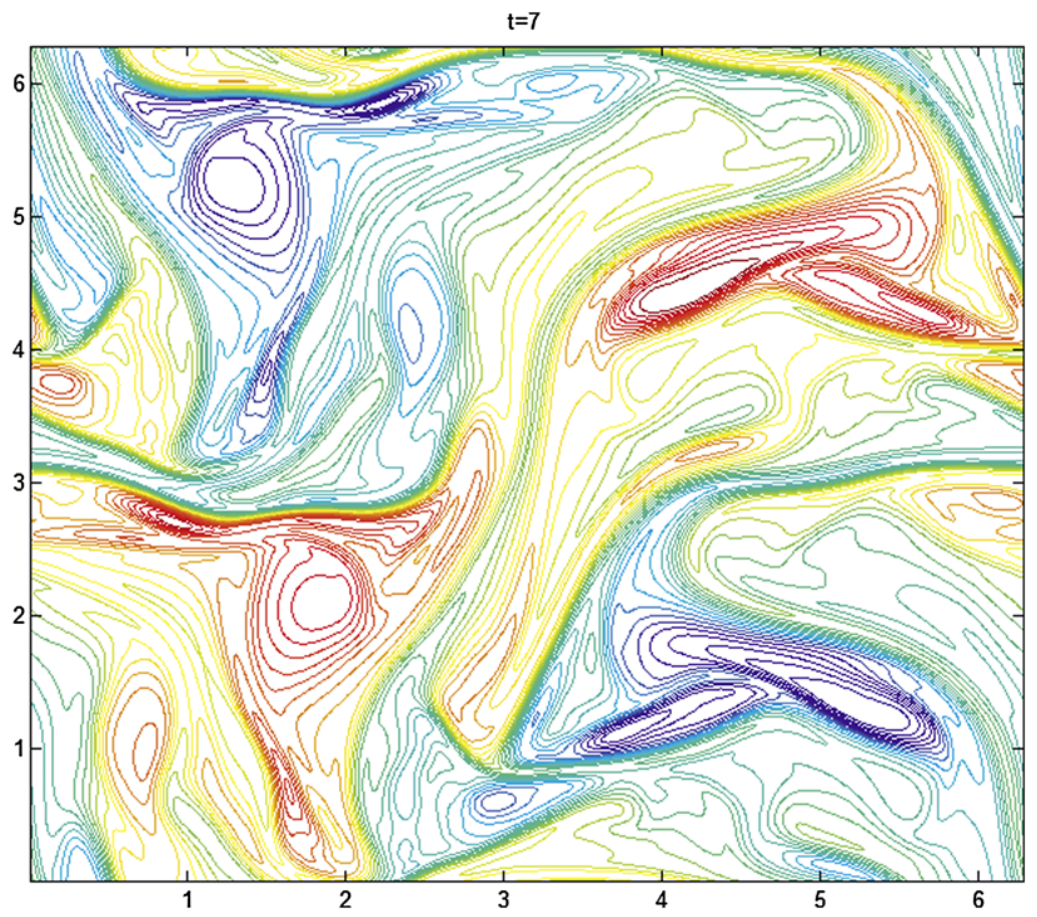

Fig. 27 Contours of $\theta$ for data in (1.6), $t=7$

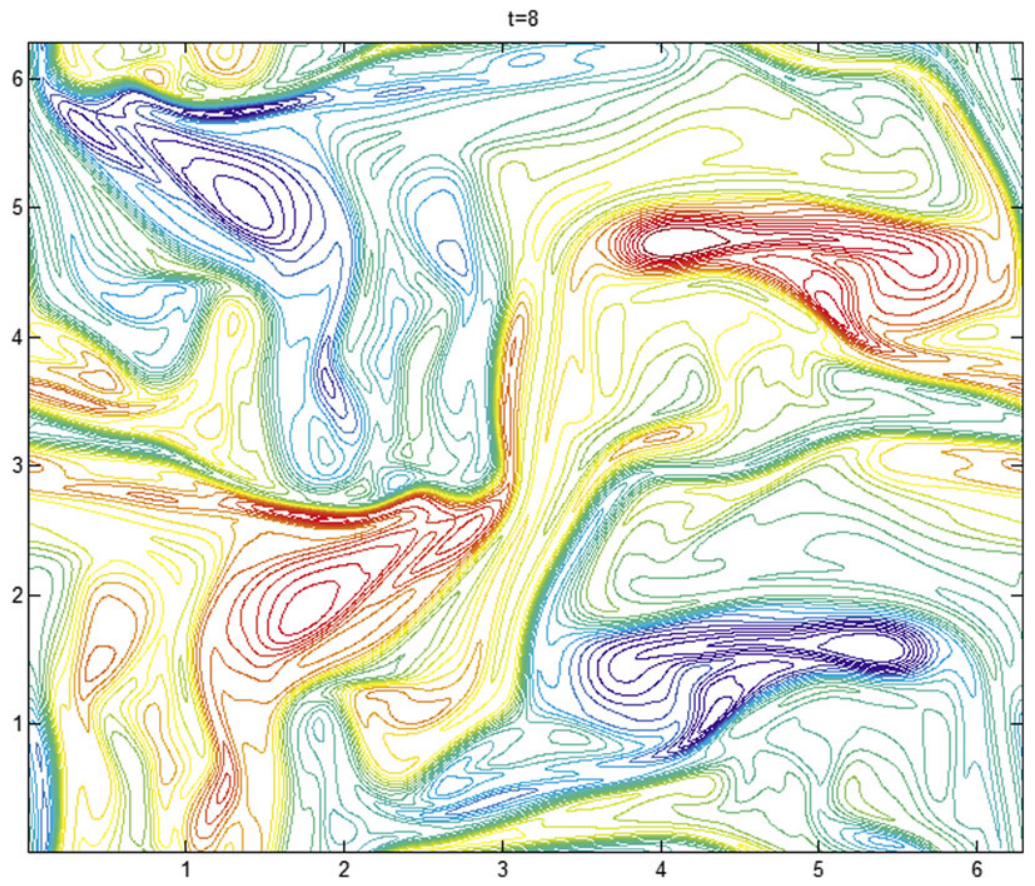

Fig. 28 Contours of $\theta$ for data in (1.6), $t=8$ 

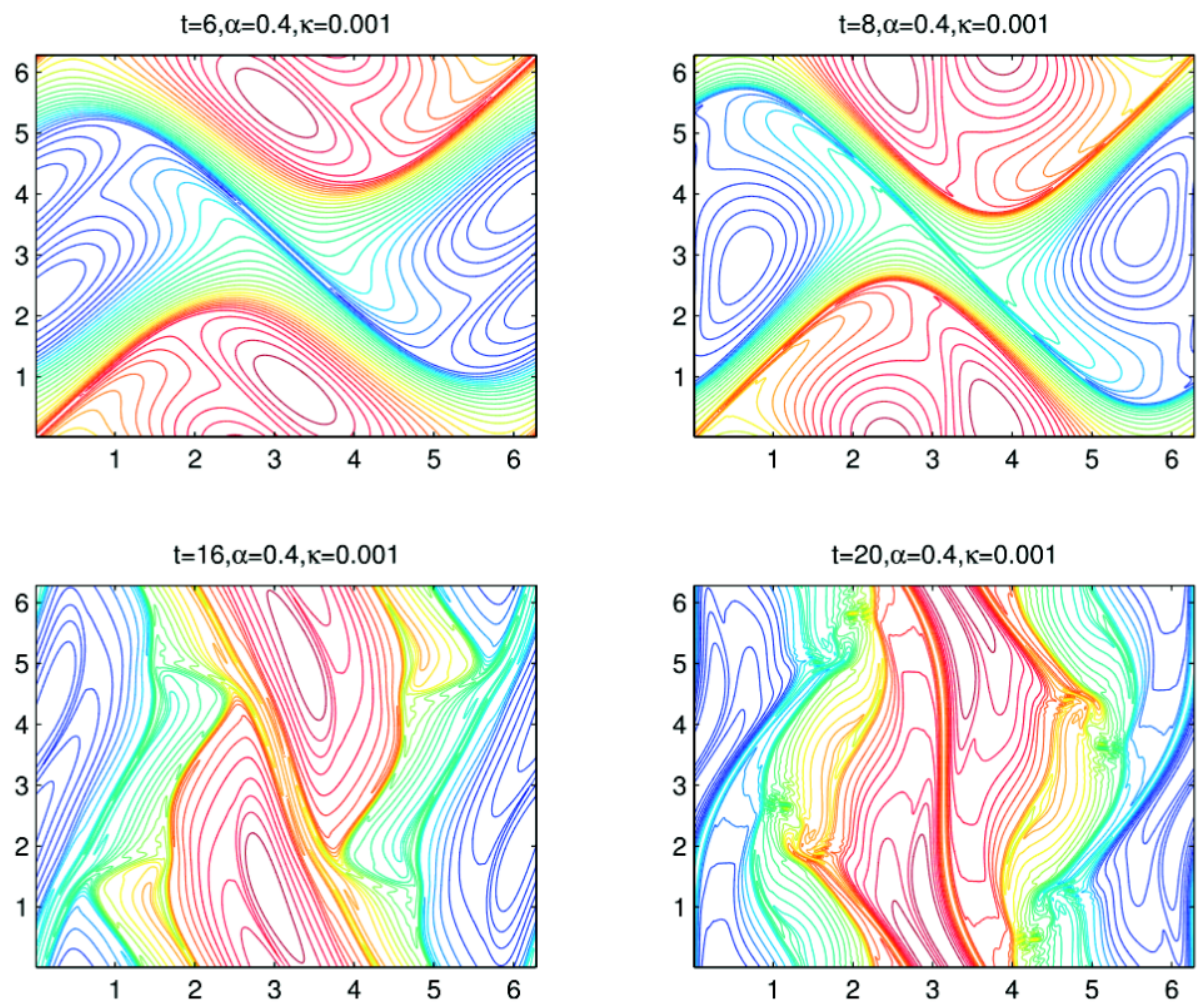

Fig. 29 Contours of $\theta, \alpha=0.4, \kappa=0.001$ for data in (1.4), $N=2048, t=6,8,16,20$

The third set of experiments is designed to understand the role of $\kappa$ in the global regularity issue. For a large value of $\kappa$, say $\kappa=0.05$, the solution becomes smooth very quickly and appears to approach a steady-state solution for large time (see Fig. 31). As we decrease $\kappa$, the solutions begin to exhibit richer structures for some finite-time and start to approach steady-states for large time. As an illustration, we provide the plots for $\kappa=0.01$ (see Fig. 32).

\section{Conclusion}

We performed a careful numerical study of the evolution of solutions of the SQG and dissipative SQG equations, beyond the previously calculated times. In the case of the SQG equation, we confirmed the formation of a strong hyperbolic saddle front at about time 7.5, observed in previous calculations [15]. We found that the hyperbolic saddle is followed by a steep anti-parallel double front, while the maximum gradient continues to grow, up to about $t=13.5$. The gradient decays afterwards and no regeneration of strong fronts is observed, although the weakened anti-parallel double front persists for a long time. In the dissipative SQG case, there is no evidence of critical behavior at $\alpha=\frac{1}{2}$, the solutions are smooth, and, for moderate generalized diffusivity $\kappa$, they easily reach steadystate. 
$\mathrm{t}=14, \alpha=0.45, \kappa=0.001$, maxgradtheta $=5.3469$

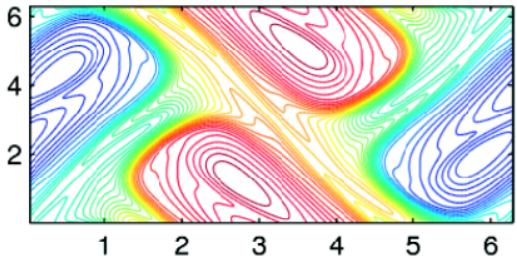

$\mathrm{t}=14, \alpha=0.49, \kappa=0.001$, maxgradtheta $=5.1820$

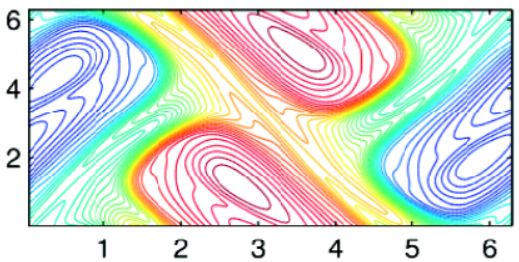

$\mathrm{t}=14, \alpha=0.48, \kappa=0.001$, maxgradtheta $=5.2239$

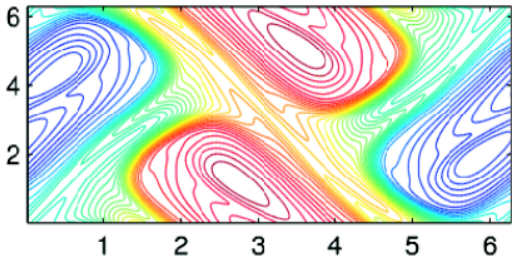

$\mathrm{t}=14, \alpha=0.5, \kappa=0.001$, axgradtheta $=5.1311$

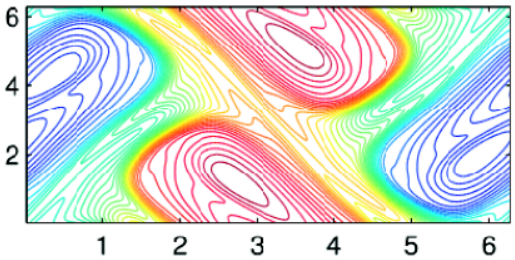

$\mathrm{t}=14, \alpha=0.51, \kappa=0.001$, maxgradtheta $=5.0937$

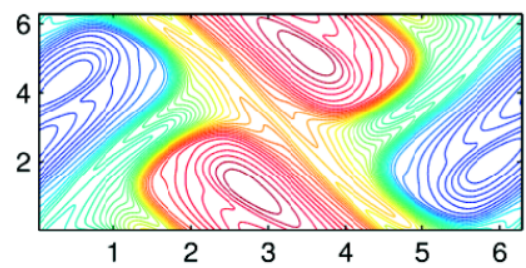

Fig. 30 Comparison of contours for $\alpha$ near $0.5(\kappa=0.001$ for data in (1.4), $N=2048, t=14)$

Fig. 31 Contours of $\theta, \kappa=0.05$, $\alpha=0.4$ for data in (1.4), $N=2048, t$ from 5 to 30
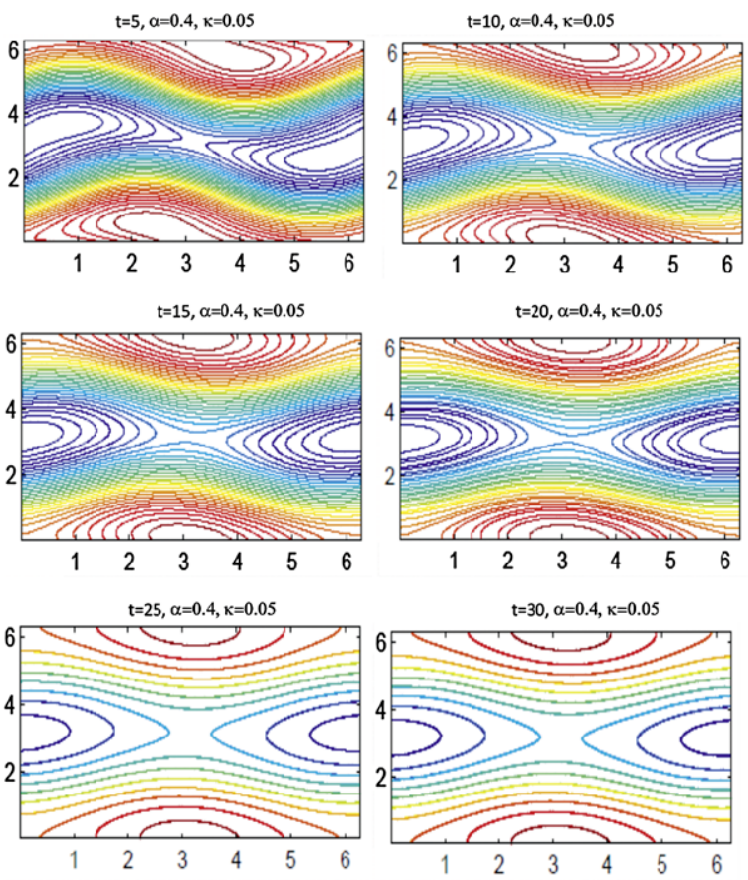


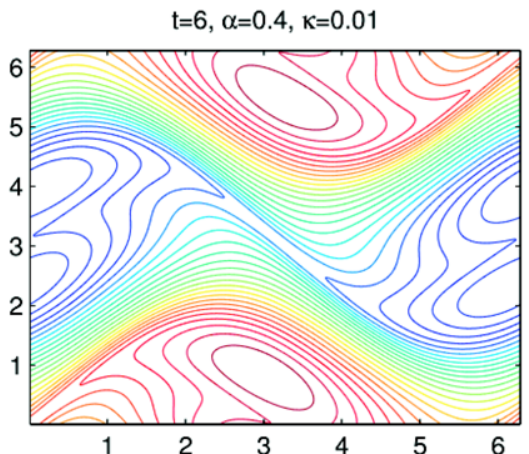

$\mathrm{t}=50, \alpha=0.4, \kappa=0.01$

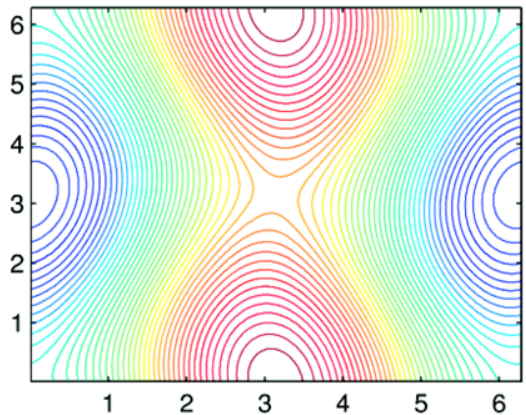

$\mathrm{t}=12, \alpha=0.4, \kappa=0.01$

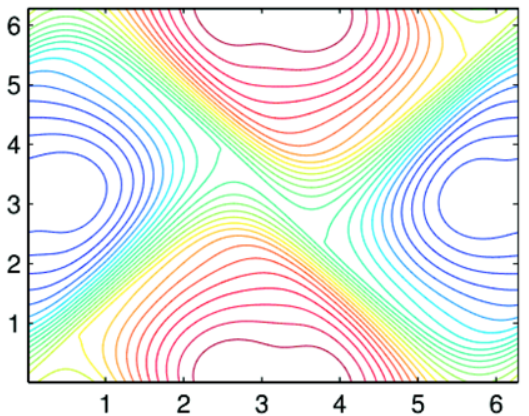

$\mathrm{t}=100, \alpha=0.4, \kappa=0.01$

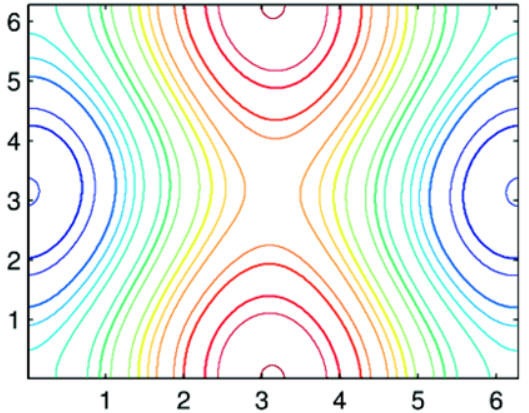

Fig. 32 Contours of $\theta, \kappa=0.01, \alpha=0.4$ for data in (1.4), $N=2048, t=6,12,50,100$

Acknowledgements Constantin is partially supported by a NSF grant. Sharma and Wu would like to thank Professors Thomas Hou, Koji Ohkitani and Yanqiu Wang for advice, and thank Dr. Dana Brunson of OSU High Performance Computing Center and Professor Henry Neeman of OU Supercomputing Center for help and support. Sharma and Wu are partially supported by NSF grant DMS 0907913. Wu also acknowledges the support of the AT\&T Foundation at OSU and the hospitality of Applied Mathematics Department at National Chiao Tung University, Taiwan.

\section{References}

1. Abidi, H., Hmidi, T.: On the global well-posedness of the critical quasi-geostrophic equation. SIAM J. Math. Anal. 40, 167-185 (2008)

2. Blumen, W.: Uniform potential vorticity flow, Part I. Theory of wave interactions and two-dimensional turbulence. J. Atmos. Sci. 35, 774-783 (1978)

3. Caffarelli, L., Silvestre, L.: An extension problem related to the fractional Laplacian. Commun. Partial Differ. Equ. 32, 1245-1260 (2007)

4. Caffarelli, L., Vasseur, A.: Drift diffusion equations with fractional diffusion and the quasi-geostrophic equation. Ann. Math. 171, 1903-1930 (2010)

5. Carrillo, J., Ferreira, L.: The asymptotic behaviour of subcritical dissipative quasi-geostrophic equations. Nonlinearity 21, 1001-1018 (2008)

6. Chae, D.: The quasi-geostrophic equation in the Triebel-Lizorkin spaces. Nonlinearity 16, 479-495 (2003)

7. Chae, D.: On the regularity conditions for the dissipative quasi-geostrophic equations. SIAM J. Math. Anal. 37, 1649-1656 (2006)

8. Chae, D.: The geometric approaches to the possible singularities in the inviscid fluid flows. J. Phys. A 41, 365501 (2008), 11 p. 
9. Chae, D., Lee, J.: Global well-posedness in the super-critical dissipative quasi-geostrophic equations. Commun. Math. Phys. 233, 297-311 (2003)

10. Chen, Q., Miao, C., Zhang, Z.: A new Bernstein's inequality and the 2D dissipative quasi-geostrophic equation. Commun. Math. Phys. 271, 821-838 (2007)

11. Chen, Q., Zhang, Z.: Global well-posedness of the 2D critical dissipative quasi-geostrophic equation in the Triebel-Lizorkin spaces. Nonlinear Anal. 67, 1715-1725 (2007)

12. Constantin, P.: Euler equations, Navier-Stokes equations and turbulence. In: Mathematical Foundation of Turbulent Viscous Flows. Lecture Notes in Math., vol. 1871, pp. 1-43. Springer, Berlin (2006)

13. Constantin, P., Córdoba, D., Wu, J.: On the critical dissipative quasi-geostrophic equation. Indiana Univ. Math. J. 50, 97-107 (2001)

14. Constantin, P., Iyer, G., Wu, J.: Global regularity for a modified critical dissipative quasi-geostrophic equation. Indiana Univ. Math. J. 57, 2681-2692 (2008)

15. Constantin, P., Majda, A., Tabak, E.: Formation of strong fronts in the 2-D quasi-geostrophic thermal active scalar. Nonlinearity 7, 1495-1533 (1994)

16. Constantin, P., Nie, Q., Schörghofer, N.: Nonsingular surface quasi-geostrophic flow. Phys. Lett. A 241, 168-172 (1998)

17. Constantin, P., Wu, J.: Behavior of solutions of 2D quasi-geostrophic equations. SIAM J. Math. Anal. 30, 937-948 (1999)

18. Constantin, P., Wu, J.: Regularity of Hölder continuous solutions of the supercritical quasi-geostrophic equation. Ann. Inst. Henri Poincaré, Anal. Non Linéaire 25, 1103-1110 (2008)

19. Constantin, P., Wu, J.: Hölder continuity of solutions of supercritical dissipative hydrodynamic transport equation. Ann. Inst. Henri Poincaré, Anal. Non Linéaire 26, 159-180 (2009)

20. Córdoba, D.: Nonexistence of simple hyperbolic blow-up for the quasi-geostrophic equation. Ann. Math. 148, 1135-1152 (1998)

21. Córdoba, A., Córdoba, D.: A maximum principle applied to quasi-geostrophic equations. Commun. Math. Phys. 249, 511-528 (2004)

22. Córdoba, D., Fefferman, Ch.: Behavior of several two-dimensional fluid equations in singular scenarios. Proc. Natl. Acad. Sci. USA 98, 4311-4312 (2001)

23. Córdoba, D., Fefferman, Ch.: Scalars convected by a two-dimensional incompressible flow. Commun. Pure Appl. Math. 55, 255-260 (2002)

24. Córdoba, D., Fefferman, Ch.: Growth of solutions for QG and 2D Euler equations. J. Am. Math. Soc. 15, 665-670 (2002)

25. Córdoba, D., Fontelos, M., Mancho, A., Rodrigo, J.: Evidence of singularities for a family of contour dynamics equations. Proc. Natl. Acad. Sci. USA 102, 5949-5952 (2005)

26. Deng, J., Hou, T.Y., Li, R., Yu, X.: Level set dynamics and the non-blowup of the 2D quasi-geostrophic equation. Methods Appl. Anal. 13, 157-180 (2006)

27. Dong, B., Chen, Z.: Asymptotic stability of the critical and super-critical dissipative quasi-geostrophic equation. Nonlinearity 19, 2919-2928 (2006)

28. Dong, H., Du, D.: Global well-posedness and a decay estimate for the critical dissipative quasigeostrophic equation in the whole space. Discrete Contin. Dyn. Syst. 21, 1095-1101 (2008)

29. Dong, H., Li, D.: Finite time singularities for a class of generalized surface quasi-geostrophic equations. Proc. Am. Math. Soc. 136, 2555-2563 (2008)

30. Dong, H., Li, D.: Spatial analyticity of the solutions to the subcritical dissipative quasi-geostrophic equations. Arch. Ration. Mech. Anal. 189, 131-158 (2008)

31. Dong, H., Pavlovic, N.: A regularity criterion for the dissipation quasi-geostrophic equation. Ann. Inst. Henri Poincaré, Anal. Non Linéaire 26, 1607-1619 (2009)

32. Dong, H., Pavlovic, N.: Regularity criteria for the dissipative quasi-geostrophic equations in Holder spaces. Commun. Math. Phys. 290, 801-812 (2009)

33. Gill, A.E.: Atmosphere-Ocean Dynamics. Academic Press, San Diego (1982)

34. Gottlieb, D., Orszag, S.A.: Numerical Analysis of Spectral Methods: Theory and Applications. CBMSNSF Regional Conference Series in Applied Mathematics, vol. 26. SIAM, Philadelphia (1977)

35. Held, I., Pierrehumbert, R., Garner, S., Swanson, K.: Surface quasi-geostrophic dynamics. J. Fluid Mech. 282, 1-20 (1995)

36. Hmidi, T., Keraani, S.: Global solutions of the super-critical 2D quasi-geostrophic equation in Besov spaces. Adv. Math. 214, 618-638 (2007)

37. Hou, T.Y., Li, R.: Computing nearly singular solutions using pseudo-spectral methods. J. Comput. Phys. 226, 379-397 (2007)

38. Ju, N.: The maximum principle and the global attractor for the dissipative 2D quasi-geostrophic equations. Commun. Math. Phys. 255, 161-181 (2005)

39. Ju, N.: Geometric constrains for global regularity of 2D quasi-geostrophic flows. J. Differ. Equ. 226, 54-79 (2006) 
40. Kiselev, A.: Some recent results on the critical surface quasi-geostrophic equation: a review, preprint

41. Kiselev, A., Nazarov, F., Volberg, A.: Global well-posedness for the critical 2D dissipative quasigeostrophic equation. Invent. Math. 167, 445-453 (2007)

42. Lemarie-Rieusset, P.-G.: Recent Developments in the Navier-Stokes Problem. Chapman \& Hall/CRC, Boca Raton (2002)

43. Li, D.: Existence theorems for the 2D quasi-geostrophic equation with plane wave initial conditions. Nonlinearity 22, 1639-1651 (2009)

44. Li, D., Rodrigo, J.: Blow up for the generalized surface quasi-geostrophic equation with supercritical dissipation. Commun. Math. Phys. 286, 111-124 (2009)

45. Majda, A.: Introduction to PDEs and Waves for the Atmosphere and Ocean. Courant Lecture Notes, vol. 9. Courant Institute of Mathematical Sciences and American Mathematical Society, New York (2003)

46. Majda, A., Tabak, E.: A two-dimensional model for quasigeostrophic flow: comparison with the twodimensional Euler flow. Physica D 98, 515-522 (1996)

47. Marchand, F.: Propagation of Sobolev regularity for the critical dissipative quasi-geostrophic equation. Asymptot. Anal. 49, 275-293 (2006)

48. Marchand, F.: Existence and regularity of weak solutions to the quasi-geostrophic equations in the spaces $L^{p}$ or $\dot{H}^{-1 / 2}$. Commun. Math. Phys. 277, 45-67 (2008)

49. Marchand, F.: Weak-strong uniqueness criteria for the critical quasi-geostrophic equation. Physica D 237, 1346-1351 (2008)

50. Marchand, F., Lemarié-Rieusset, P.G.: Solutions auto-similaires non radiales pour l'équation quasigéostrophique dissipative critique. C. R. Math. Acad. Sci. Paris 341, 535-538 (2005)

51. Miura, H.: Dissipative quasi-geostrophic equation for large initial data in the critical Sobolev space. Commun. Math. Phys. 267, 141-157 (2006)

52. Ohkitani, K., Yamada, M.: Inviscid and inviscid-limit behavior of a surface quasigeostrophic flow. Phys. Fluids 9, 876-882 (1997)

53. Okitani, K., Sakajo, T.: Oscillatory damping in long-time evolution of the surface quasi-geostrophic equations with generalized viscosity: a numerical study, preprint

54. Pedlosky, J.: Geophysical Fluid Dynamics. Springer, New York (1987)

55. Resnick, S.: Dynamical problems in nonlinear advective partial differential equations. Ph.D. thesis, University of Chicago (1995)

56. Rodrigo, J.: The vortex patch problem for the surface quasi-geostrophic equation. Proc. Natl. Acad. Sci. USA 101, 2684-2686 (2004)

57. Rodrigo, J.: On the evolution of sharp fronts for the quasi-geostrophic equation. Commun. Pure Appl. Math. 58, 821-866 (2005)

58. Schonbek, M., Schonbek, T.: Asymptotic behavior to dissipative quasi-geostrophic flows. SIAM J. Math. Anal. 35, 357-375 (2003)

59. Schonbek, M., Schonbek, T.: Moments and lower bounds in the far-field of solutions to quasi-geostrophic flows. Discrete Contin. Dyn. Syst. 13, 1277-1304 (2005)

60. Wu, J.: Quasi-geostrophic-type equations with initial data in Morrey spaces. Nonlinearity 10, 1409-1420 (1997)

61. Wu, J.: Inviscid limits and regularity estimates for the solutions of the 2-D dissipative quasi-geostrophic equations. Indiana Univ. Math. J. 46, 1113-1124 (1997)

62. Wu, J.: Dissipative quasi-geostrophic equations with $L^{p}$ data. Electron. J. Differ. Equ. 2001, 1-13 (2001)

63. Wu, J.: The quasi-geostrophic equation and its two regularizations. Commun. Partial Differ. Equ. 27, 1161-1181 (2002)

64. Wu, J.: The generalized incompressible Navier-Stokes equations in Besov spaces. Dyn. Partial Differ. Equ. 1, 381-400 (2004)

65. Wu, J.: Global solutions of the 2D dissipative quasi-geostrophic equation in Besov spaces. SIAM J. Math. Anal. 36, 1014-1030 (2004/2005)

66. Wu, J.: The quasi-geostrophic equation with critical or supercritical dissipation. Nonlinearity 18, 139154 (2005)

67. Wu, J.: Solutions of the 2-D quasi-geostrophic equation in Hölder spaces. Nonlinear Anal. 62, 579-594 (2005)

68. Wu, J.: Lower bounds for an integral involving fractional Laplacians and the generalized Navier-Stokes equations in Besov spaces. Commun. Math. Phys. 263, 803-831 (2006)

69. $\mathrm{Wu}, \mathrm{J} .:$ Existence and uniqueness results for the 2-D dissipative quasi-geostrophic equation. Nonlinear Anal. 67, 3013-3036 (2007)

70. Yu, X.: Remarks on the global regularity for the super-critical 2D dissipative quasi-geostrophic equation. J. Math. Anal. Appl. 339, 359-371 (2008) 
71. Zhang, Z.: Well-posedness for the 2D dissipative quasi-geostrophic equations in the Besov space. Sci. China Ser. A 48, 1646-1655 (2005)

72. Zhang, Z.: Global well-posedness for the $2 \mathrm{D}$ critical dissipative quasi-geostrophic equation. Sci. China Ser. A 50, 485-494 (2007)

73. Zhou, Y.: Decay rate of higher order derivatives for solutions to the 2-D dissipative quasi-geostrophic flows. Discrete Contin. Dyn. Syst. 14, 525-532 (2006) 